\title{
Pangenome analysis of Bifidobacterium longum and site-directed mutagenesis through by-pass of restriction-modification systems
}

\author{
A. O'Callaghan, F. Bottacini, M. O'Connell Motherway and D. van Sinderen*
}

\begin{abstract}
Background: Bifidobacterial genome analysis has provided insights as to how these gut commensals adapt to and persist in the human GIT, while also revealing genetic diversity among members of a given bifidobacterial (sub)species. Bifidobacteria are notoriously recalcitrant to genetic modification, which prevents exploration of their genomic functions, including those that convey (human) health benefits.

Methods: PacBio SMRT sequencing was used to determine the whole genome seqeunces of two $B$. longum subsp. longum strains. The B. longum pan-genome was computed using PGAP v1.2 and the core B. longum phylogenetic tree was constructed using a maximum-likelihood based approach in PhyML v3.0. M.blmNCIl was cloned in E. coli and an internal fragment if arfBarfB was cloned into pORI19 for insertion mutagenesis.

Results: In this study we present the complete genome sequences of two Bifidobacterium longum subsp. longum strains. Comparative analysis with thirty one publicly available $B$. longum genomes allowed the definition of the $B$. longum core and dispensable genomes. This analysis also highlighted differences in particular metabolic abilities between members of the $B$. longum subspecies infantis, longum and suis. Furthermore, phylogenetic analysis of the $B$. longum core genome indicated the existence of a novel subspecies. Methylome data, coupled to the analysis of restriction-modification systems, allowed us to substantially increase the genetic accessibility of $B$. longum subsp. longum NCIMB 8809 to a level that was shown to permit site-directed mutagenesis.

Conclusions: Comparative genomic analysis of thirty three B. longum representatives revealed a closed pan-genome for this bifidobacterial species. Phylogenetic analysis of the B. longum core genome also provides evidence for a novel fifth $B$. longum subspecies. Finally, we improved genetic accessibility for the strain B. longum subsp. longum NCIMB 8809 , which allowed the generation of a mutant of this strain.
\end{abstract}

Keywords: Bifidobacterium longum, Comparative genomics, Pan-genome, Probiotics, Restriction modification systems, Methylation

\section{Background}

Bifidobacteria have been isolated from several ecological niches linked to the gastro intestinal tract (GIT) of animals, including the human GIT, where they represent prominent members of the gut microbiota [1, 2]. Bifidobacteria have attracted significant scientific and commercial interest due to their purported health-promoting or probiotic effects conferred to their (human) host, such as strengthening/maintenance of the intestinal

\footnotetext{
* Correspondence: d.vansinderen@ucc.ie

APC Microbiome Institute \& School of Microbiology, University College Cork, Western Road, Cork, Ireland
}

barrier, modulation of the immune system, and pathogen exclusion $[3,4]$.

Fourty eight different bifidobacterial species are currently recognised, including nine subspecies, and of these only fifteen species are represented by fully assembled genome sequences, of which there are currently fifty four publicly available (June 2015, source: http://www.ncbi.nlm.nih.gov/ Taxonomy/Browser/wwwtax.cgi). Multiple genome sequences are available for certain bifidobacteiral species allowing for pan-genome analysis, which is the total number of different genes encoded by a certain species [5]. Recent studies have deduced the pan-genome 
for Bifidobacterium breve [6] and Bifidobacterium animalis subsp. lactis [7], while another focussed on the genomic diversity of Bifidobacterium adolescentis [8]. In order to map the evolutionary development of the Bifidobacterium genus, an extensive comparative study was recently performed on individual representatives of 47 bifidobacterial (sub)species [9, 10]. One of these comparative studies suggests that bifidobacteria and their animal hosts co-evolved, and that this co-evolution was facilitated by both gene loss and acquisition events to allow for (sub)species-specific adaptations to a glycan-rich environment [9].

Functional genome analysis of bifidobacteria is important in order to understand how this species adapts to a particular niche. For example, more than $8 \%$ of the annotated genes found in the genomes of B. longum and $B$. breve are predicted to encode proteins involved in the metabolism of complex plant-derived carbohydrates [11]. In contrast, the human genome is predicted to encode just seventeen enzymes involved in glycan catabolism $[12,13]$. It is believed that this paucity is compensated by the metabolic abilities provided by the gut microbiota, including bifidobacteria, thus allowing the (human) host to (indirectly) digest complex polysaccharides that would otherwise be deemed non-digestible. It is therefore important to obtain an in depth understanding of carbohydrate utilisation by gut commensals and its impact on their host.

Despite the generally held view that bifidobacteria elicit positive health effects on their host, the underlying molecular mechanisms are as yet far from fully understood [4]. One of the key reasons for this knowledge gap is the difficulty in genetically modifying bifidobacteria, in part attributed to the presence of restriction-modification (R-M) systems [4, 14], which provide a powerful and natural defence for prokaryotic cells against invading foreign DNA, in particular bacteriophages [15]. R-M systems are currently classified into four well characterised types, I, II, III and IV, based on their co-factor requirements, protein composition, and target/cleavage sequence characteristics [16]. Type I R-M systems consist of three subunits that are responsible for methylation, specificity and restriction, respectively, and recognise asymmetric sequences that comprise of two components that are separated by a non-specific spacer. Type I R-M systems require $S$-Adenosylmethionine (AdoMet), ATP and $\mathrm{Mg}^{2+}$, typically methylate adenine residues and cut unmodified DNA at sites distal to the recognition sites [16]. Classical type II R-M systems recognise palindromic sequences of 4-8 bp in length and cut DNA into discrete fragments within or close to the recognition site [16]. The type II RM methyltransferase (MTase) modifies adenosyl or cytosyl residues of a particular recognition sequence, which, when unmethylated, is recognised and cut by the corresponding restriction endonuclease (REase) [17, 18]. Type III R-M systems consists of two subunits that are responsible for DNA recognition and modification (Mod subunit), and DNA cleavage (Res subunit). Type III R-M systems recognise inversely orientated asymmetric DNA sequences, where the Res subunit cuts the DNA close to one of these recognition sites [19]. Finally, type IV restriction enzymes differ from those previously described as they recognise and cleave DNA only when the recognition site is methylated [18].

Independent studies in bifidobacteria have demonstrated that following modification of plasmid DNA, R-M systems can be by-passed, thereby resulting in a substantial increase in transformation efficiency and in some cases the successful generation of mutants $[15,20]$. In a recent study, data gained from methylome analysis led to a moderate improvement in the transformation efficiency of the strain Bifidobacterium animalis subsp. lactis CNCM I-2494 [21]. However, overcoming R-M systems of a given species is but one tool of the expanding bifidobacterial genetic tool box, which now includes conjugationbased methods [22, 23], a temperature-sensitive plasmid [24] and a double-crossover, marker-less gene deletion system [25].

In this study we present the complete genome sequence for B. longum subsp. longum NCIMB 8809 and B. longum subsp. longum CCUG 30698, and associated methylome and R-M analyses. Comparative analysis of the genomes of these two strains with publicly available complete and incomplete $B$. longum genomes enabled us to explore the genomic diversity among members of the B. longum subspecies longum, infantis and suis. In addition, by exploiting methylome and genomic data analysis, we were able to assess the functionality of the R-M systems native to $B$. longum subsp. longum NCIMB 8809. This allowed us to improve the genetic accessibility of $B$. longum subsp. longum NCIMB 8809, permitting site-directed mutagenesis of this strain.

\section{Results and discussion}

\section{General features of $B$. longum genomes}

The complete genome sequence was determined for two $B$. longum subsp. longum strains that had been isolated from infant faeces (B. longum subsp. longum NCIMB 8809 ) or a human adult intestine (B. longum subsp. longum CCUG 30698). Salient details of each of these genomes are presented in Table 1 . The observed $\mathrm{G}+\mathrm{C} \%$ content of both B. longum genomes $(60.1 \% \mathrm{G}+\mathrm{C} \%$ content for B. longum subsp. longum NCIMB 8809 and $60.22 \mathrm{G}+\mathrm{C} \%$ content for B. longum subsp. longum CCUG 30698) is consistent with that reported for other bifidobacterial genomes [3]. The genome of B. longum subsp. longum CCUG 30698 contains a substantially higher number of tRNAs compared to most other sequenced $B$. longum strains, although a similarly high 
Table 1 Bifiobacterium longum general genome features

\begin{tabular}{lllll}
\hline & $\begin{array}{l}\text { B. longum subsp. longum } \\
\text { NCIMB 8809 }\end{array}$ & $\begin{array}{l}\text { B. longum subsp. longum } \\
\text { CCUG 30698 }\end{array}$ & $\begin{array}{l}\text { B. longum subsp. longum } \\
\text { NCC2705 }\end{array}$ & $\begin{array}{l}\text { B. longum subsp. longum } \\
\text { DJO10A }\end{array}$ \\
\hline Isolated from & Nursling stool & Human adult intestine & Infant faeces & Young adult faeces \\
Genome Size & 2.34 & 2.45 & 2.25 & 2.37 \\
G + C content \% & 60.1 & 60.22 & 60.12 & 60.15 \\
Number of identified genes & 1872 & 1983 & 1727 & 1990 \\
Percentage of genes functionally assigned & $77 \%$ & $74 \%$ & $79 \%$ & $76 \%$ \\
Prophage & 1 (complete) & 1 & 1 & 1 \\
Episome & 1 & 0 & 1 & 1 \\
rRNA & 3 & 2 & 4 & 4 \\
tRNA & 56 & 70 & 57 & 58 \\
CRISPR & 0 & 0 & 1 & 1 \\
\hline
\end{tabular}

number of tRNA-encoding elements is present in the genome of strain B. longum subsp. infantis ATCC 15697 [26]. BLASTP analyses of deduced proteins of all identified ORFs in both $B$. longum genomes was performed against the Cluster of Orthologous Groups (COG) database and the obtained results show that a high percentage of predicted proteins is dedicated to general cellular housekeeping functions including amino acid transport and metabolism, and carbohydrate transport and metabolism (10.6\% in the case of B. longum subsp. longum NCIMB

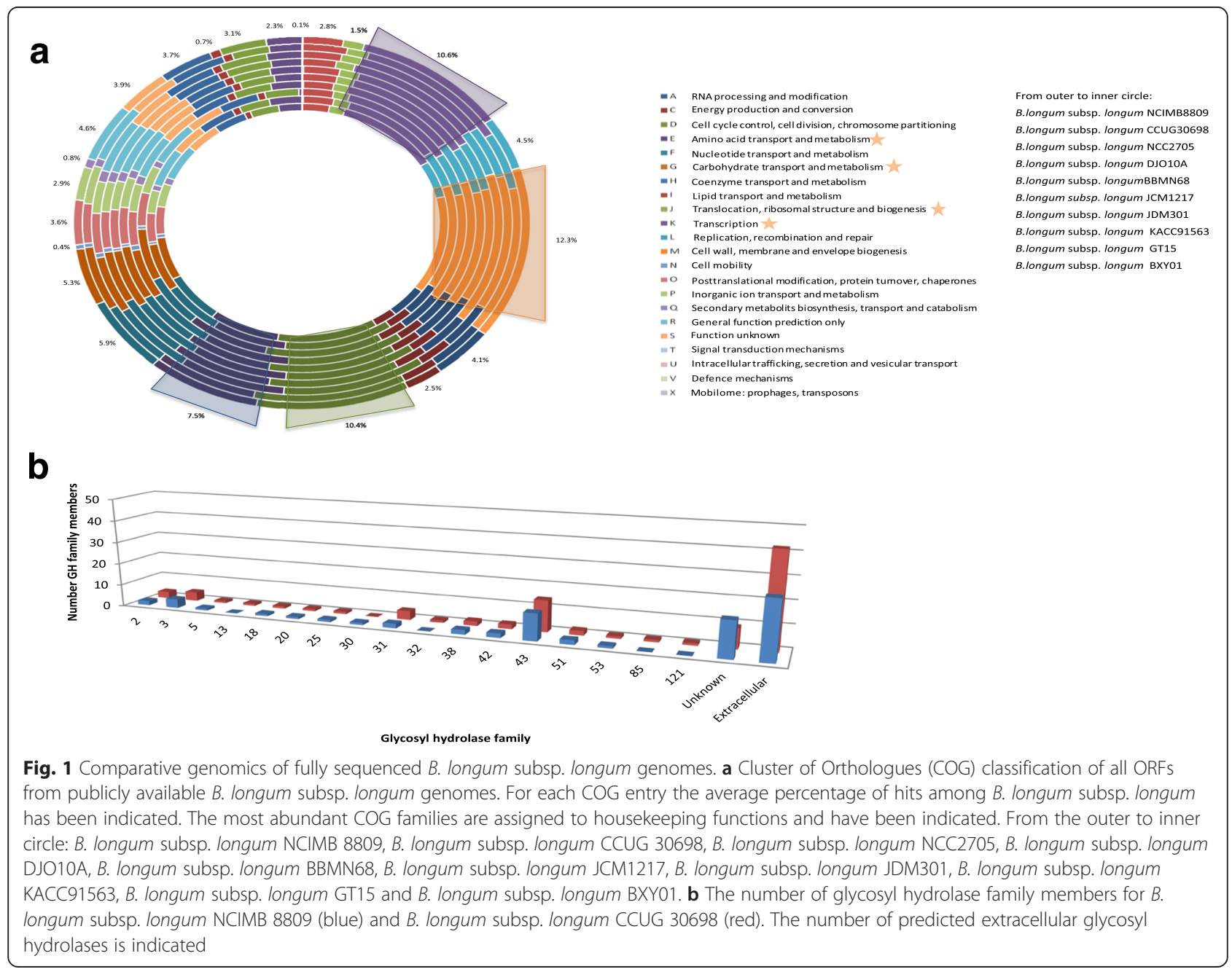


8809 and $12.3 \%$ in the case of B. longum subsp. longum CCUG 30698). These percentages are consistent with those previously observed for other bifidobacterial genomes [6, 27-29] (Fig. 1, panel a).

Further exploration of the sequence data revealed that both genomes encode a single homologous phosphoenolpyruvate-phosphotransferase system (PEPPTS). This system exhibits $34 \%$ identity across $99 \%$ of the query sequence to a fructose-specific PEP-PTS system encoded by B. breve UCC2003 [30]. In relation to carbohydrate-active enzymes, 35 glycosyl hydrolases (GHs) were identified in B. longum subsp. longum NCIMB 8809, whereas B. longum subsp. longum CCUG 30698 is predicted to encode 40 GHs (Fig. 1, panel b). The B. longum subsp. longum NCIMB 8809 genome specifies GHs that belong to thirteen different $\mathrm{GH}$ families, while the $B$. longum subsp. longum CCUG 30698 genome is predicted to specify GHs that are from fifteen different $\mathrm{GH}$ families. Interestingly, both genomes are predicted to encode a high number of GHs that belong to the GH family 43. B. longum subsp. longum NCIMB 8809 is predicted to encode twelve members of GH family 43 , seven of which are predicted to be extracellular (Fig. 1, panel b). $B$. longum subsp. longum CCUG 30698 on the other hand is predicted to encode fourteen GH43 family members of the, of which nine are predicted to be extracellular (Fig. 1, panel b). Many members of the GH43 family represent inverting enzymes active against long-chain carbohydrates (polysaccharides), which are typically constituents of plant cell walls and represent so-called non-digestible dietary fibers, such as arabinoxylan, arabinan, galactan and xylan [31]. Such predicted plant polysaccharide-degrading activities had previously been described for the $B$. longum subsp. longum NCC2705 genome, when it was reported that, according to COG functional classification, more than $8.5 \%$ of the predicted proteins encoded by this strain were associated with carbohydrate metabolism and transport.

\section{Synteny and variability of $B$. longum subsp. longum genomes}

To investigate the syntenic relationship between the two newly sequenced $B$. longum genomes, dotplot comparisons were constructed using B. longum subsp. longum NCC2705 as the reference genome. The resulting dotplots between the genomes of $B$. longum subsp. longum NCC2705 and B. longum subsp. longum NCIMB 8809 reveal a near continuous straight line indicating a high level of synteny (Additional file 1: Figure S1). The comparison between B. longum subsp. longum NCC2705 and B. longum subsp. longum CCUG 30698 reveals a break in genome synteny due to the presence of an apparent DNA inversion. This inversion concerns a 550,317 bp region on the B. longum subsp. longum CCUG 30698 genome. Examination of this CCUG 30698 region by PCR confirmed that this DNA inversion is genuine, and apparently stable, thus representing a distinctive feature of this genome (data not shown).

Various extracellular structures encoded by bifidobacteria have been associated with host colonisation and gut persistence, interaction with the host immune system [32-34], such as pili or fimbriae and surface-associated EPS (sEPS) [32, 34]. B. longum subsp. longum NCIMB 8809 and B. longum subsp. longum CCUG 30698 also contain genetic information for such extracellular structures (Table 2). The individual sEPS clusters identified in B. longum subsp. longum NCIMB 8809 and B. longum subsp. longum CCUG 30698 appear to lack one or more critical functions: the sEPS-specifying cluster in B. longum subsp. longum NCIMB 8809 encodes a single glycosyl transferase (GT) (a predicted priming GT), but does not contain a flippase- or other GT-encoding genes, while that of B. longum subsp. longum CCUG 30698 does not appear to encode a priming GT or polymerase (data not shown). These observations are consistent with the sedimenting phenotype of these two strains during planktonic growth (data not shown), which was also observed for a $B$. breve UCC2003-derived mutant carrying a deletion in the gene cluster responsible for sEPS production [35].

Bifidobacteria appear to be subject to regular phage attacks, events that are expected to contribute to variability within bifidobacterial genomes [36]. In light of this, searches for prophage-like elements revealed that B. longum subsp. longum NCIMB 8809 and B. longum subsp. longum CCUG 30698 harbor prophage-like elements (Table 2). The prophage-like element identified in B. longum subsp. longum NCIMB 8809 appears to be complete, whereas B. longum subsp. longum CCUG 30698 appears to harbor a single, apparently incomplete prophage-like element (due to the absence of genes that encode replication functions). Comparative analysis reveals that the prophage-like elements identified here differ from each other and that they are integrated at different locations. Although integrated at different positions within the genome, both prophage-like elements identified in B. longum subsp. longum NCIMB 8809 and B. longum subsp. longum CCUG 30698 are integrated in a tRNA ${ }^{\text {Gly }}$ gene. The genome of B. longum subsp. longum NCIMB 8809 also harbors a putative $25 \mathrm{~kb}$ episome integrated in a tRNA ${ }^{\text {Asn }}$ gene [37] (Table 2 and Additional file 2: Table S3).

\section{Pan-genome analysis}

In order to evaluate the total gene repertoire of currently sequenced representatives of the B. longum species, we applied a pan-genome analysis pipeline [38]. A total of thirty three $B$. longum genomes (complete and incomplete 
Table 2 Bifidobacterium longum variable regions that are indicated by their respective locus tags

\begin{tabular}{|c|c|c|c|c|}
\hline Variable region & $\begin{array}{l}\text { B. longum subsp. longum } \\
\text { NCIMB } 8809\end{array}$ & $\begin{array}{l}\text { B. longum subsp. longum } \\
\text { CCUG } 30698\end{array}$ & $\begin{array}{l}\text { B. longum subsp. longum } \\
\text { NCC2705 }\end{array}$ & $\begin{array}{l}\text { B. longum subsp. longum } \\
\text { DJO10A }\end{array}$ \\
\hline Prophage 1a & B8809_1609 - B8809_1668 & - & - & - \\
\hline Prophage 1b & - & BBL306_1148_BBL306_1177 & - & - \\
\hline Prophage $1 \mathrm{c}$ & - & - & - & BLD_1131 - BLD1161* \\
\hline Prophage 1d & - & - & BL0367 - BL0386 & - \\
\hline Episome 1a & B8809_0936 - B8809_1001 & - & - & - \\
\hline Episome 1b & - & - & BL1458 - BL1495 & - \\
\hline Episome 1c & - & - & - & BLD_0337 - BLD_0395 \\
\hline CRISPR & - & - & - & BLD_1903 - BLD1904 \\
\hline EPS cluster 1a & B8809_0330 - B8809_0356 & - & - & - \\
\hline EPS cluster 1b & - & BBL306_0398 - BBL306_0424 & - & - \\
\hline EPS cluster 1c & - & - & BL0225 - BL0237 & - \\
\hline EPS cluster $1 d$ & - & - & - & BLD_1565 - BLD_1579 \\
\hline EPS cluster $2 a$ & B8809_1724 - B8809_1741 & - & - & - \\
\hline EPS cluster $2 b$ & - & BBL306_1452 - BBL306_1466 & - & - \\
\hline EPS cluster 2c & - & - & BL0203 - BL0215 & - \\
\hline EPS cluster $2 d$ & - & - & - & BLD_1565 - BLD_1579 \\
\hline R/M system 1 & B8809_0606 - B8809_0607* & - & BL1473 - BL1474* & - \\
\hline R/M system 2 & B8809_0958 & - & - & - \\
\hline R/M system 3 & B8809_1350 - B8809_1355* & BBL306_1749 - BBL306_1756 * & BL1778 - BL1785* & BLD_1954 - BLD_1962* \\
\hline R/M system 4 & & BBL306_1025 & & \\
\hline R/M system 5 & - & BBL306_0230 - BBL306_0231 & - & - \\
\hline R/M system 6 & - & BBL306_0910 - BBL306_0911 & - & - \\
\hline R/M system 7 & - & - & BL0563 - BL0565 & - \\
\hline R/M system 8 & - & - & - & BLD_1565 - BLD_1579 \\
\hline pil (Sortase dependent) & B8809_1829 - B8809_1831* & BBL306_1003 - BBL306_1005* & BL0674 - BL0676-* & BLD_1467 - BLD_1469* \\
\hline pil & B8809_1607 - B8809_1608* & BBL306_1519 - BBL306_1520* & - & - \\
\hline pil (Tad) & $\begin{array}{l}\text { B8809_0107 - B8809_0112 and } \\
\text { B8809_0717** }\end{array}$ & $\begin{array}{l}\text { BBL306_0121 - BBL306_0126 and } \\
\text { BBL306_0758* }\end{array}$ & $\begin{array}{l}\text { BL0504 - BL0509 and } \\
\text { BL0879* }\end{array}$ & $\begin{array}{l}\text { BLD_0613 and BLD_1293 - } \\
\text { BLD_1298* }\end{array}$ \\
\hline
\end{tabular}

Homologous genes are indicated within the table and are marked with a "*".

genome sequences) were included in this analysis. Where relevant, genome sequences were subject to re-annotation using the same annotation procedure described previously so as to ensure that all annotations and identified ORFs were comparable (See Materials and Methods section).

The resulting pan-genome curve suggests an almost closed B. longum genome, which grows by an average of 150 genes per genome for the first thirty iterations after which the number of new gene families begins to decrease (Fig. 2, panel a). This means that, after addition of the $30^{\text {th }}$ genome, any further genome additions will result in only minor increases of the pan-genome. Analysis of the core-genome, which represents those gene families for which there is a member present in each of the strains analysed, reveals an asymptotic trend, essentially stabilizing after the $30^{\text {th }}$ genome addition at 1145 genes (Fig. 2, panel b) [38].
To identify genes that may confer a species-specific adaption, the core $B$. longum genome was compared to those of B. breve, B. bifidum, B. animalis and B. adolescentis which have been reported elsewhere $[6,7,39]$. Such differences may be a reflection of different colonisation strategies employed by the B. longum species. The most striking difference is the presence of $B$. longum-specific genes involved in the transport and metabolism of certain carbohydrates. More specifically, the $B$. longum core-genome contains genes that are predicted to encode xylanases, arabinofuranosidases, and associated $A B C$ transporters which were found to be absent in the B. breve, B. bifidum, B. animalis and $B$. adolescentis core genomes. This indicates that the conserved ability to metabolise certain, possibly xylose/ arabinose-containing plant-derived carbohydrates confers an adaptive advantage to the $B$. longum species. 

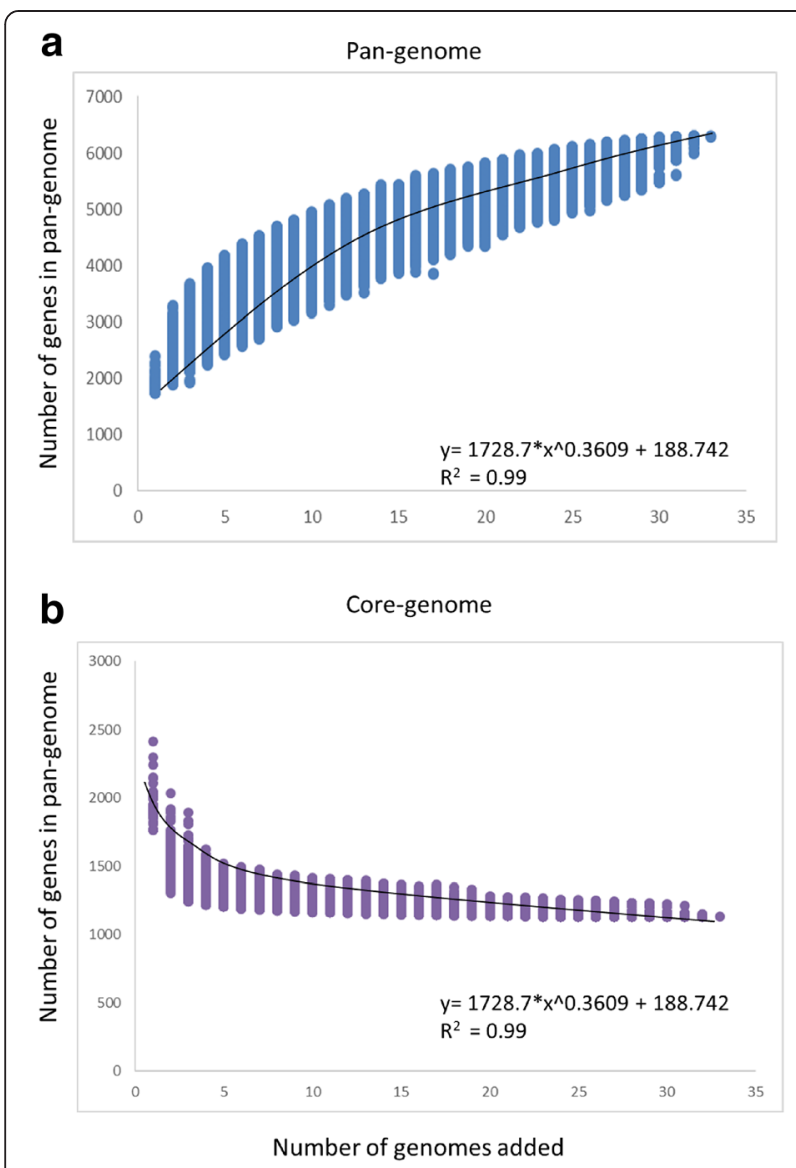

Fig. 2 Pan-genome and core-genome of B. longum. a The pan-genome plot is of the accumulated number of new genes against the number of genomes added. b The core-genome plot is represented by the accumulated number of genes attributed to the core-genomes versus the number of added genomes. The resulting functions for both plots are also reported

\section{Phylogenetic analysis of $B$. longum indicates a novel subspecies}

Up until very recently, three $B$. longum subspecies were recognized, i.e. longum, infantis and suis, although originally classified as three separate species [40-42]. The most recent classification of the three $B$. longum subspecies is summarised in Additional file 3: Table S4. This summary briefly outlines the environments from which each subspecies has been isolated, typical cell morphology of each subspecies and subspecies-specific carbohydrate fermentation patterns. A fourth subspecies, B. longum subsp. sullium, was recently recognized based on multi-locus and amplified-fragment length polymorphism approaches and urease activity, members of which had previously been classified as B. longum subsp. suis [43].

In order to analyse the phylogeny of the thirty three sequenced $B$. longum strains, which include representatives of three B. longum subspecies, a B. longum phylogenetic supertree was constructed employing the deduced protein sequences of the B. longum core-genome as conserved molecular markers (Fig. 3). A sequenced representative of the fourth B. longum subspecies was not available and so could not be included in this study. As illustrated from the resulting consensus tree, two major clades are evident (Fig. 3). The first clade consists of a group of twenty five highly related strains, the majority of which are members of the subspecies longum taxonomic group (Fig. 3; Group A). Interestingly, B. longum subsp. infantis $157 \mathrm{~F}$ and $B$. longum subsp. infantis CCUG 52486 are placed within this cluster. It is evident from the resulting consensus tree that both of these strains have been taxonomically missassigned and are in fact members of the subspecies longum.

The second clade is split into three distinctive phylogenetic groups (Fig. 3; Groups B, C and D). As expected the three (non-human) animal isolates, B. longum subsp. suis LMG21814 (isolated from porcine faeces), $B$. longum subsp. suis DSM20211 (isolated from porcine faeces) and the non-subspeciated strain B. longum AGR2137 (isolated from calf faeces) are clustered together (Fig. 3; Group D). The phylogenetic analysis performed here did not result in the identification of the subspecies sullium group, which is not surprising as no representative genome of this subspecies was available.

There is no clear separation between $B$. longum subsp. infantis ATCC 15697 and B. longum subsp. infantis EK3, which form group B, a single subspecies infantis phylogenetic group (Fig. 3; Group B). Interestingly, three strains (B. longum subsp. longum JDM301, B. longum subsp. longum CMCCP0001 and B. longum BXY01) form a separate phylogenetic group (Fig. 3; Group C), indicative of a fourth phylogenetic subgroup. Both $B$. longum subsp. longum JDM301 and B. longum BXY01 are human isolates, whereas $B$. longum subsp. longum CMCCP0001 represents a faecal isolate from a healthy infant. It is also worth noting that both B. longum subspecies longum JDM301 and B. longum subspecies longum CMCCP0001 are routinely used in commercial probiotic products $[44,45]$. B. longum subsp. longum JDM301 has been routinely grown in a stable and rich nutritional medium for a long period of time, whereas $B$. longum subsp. longum CMCCP0001 has been used in probiotic compounds for more than twenty years [44, 45]. Therefore, it is possible that this phylogenetic group has formed from strains that have evolved within industrial settings (Fig. 3; Group C). However, this cannot be established with certainty as it is unknown whether B. longum BXY01 is a commercially used strain.

Interestingly however, of all strains considered in this phylogenetic analysis and with the exception of $B$. longum subsp. longum BBMN68, the strains in Group C are the only Chinese isolates. Therefore, it is also possible that this fourth phylogenetic subgroup may represent a 


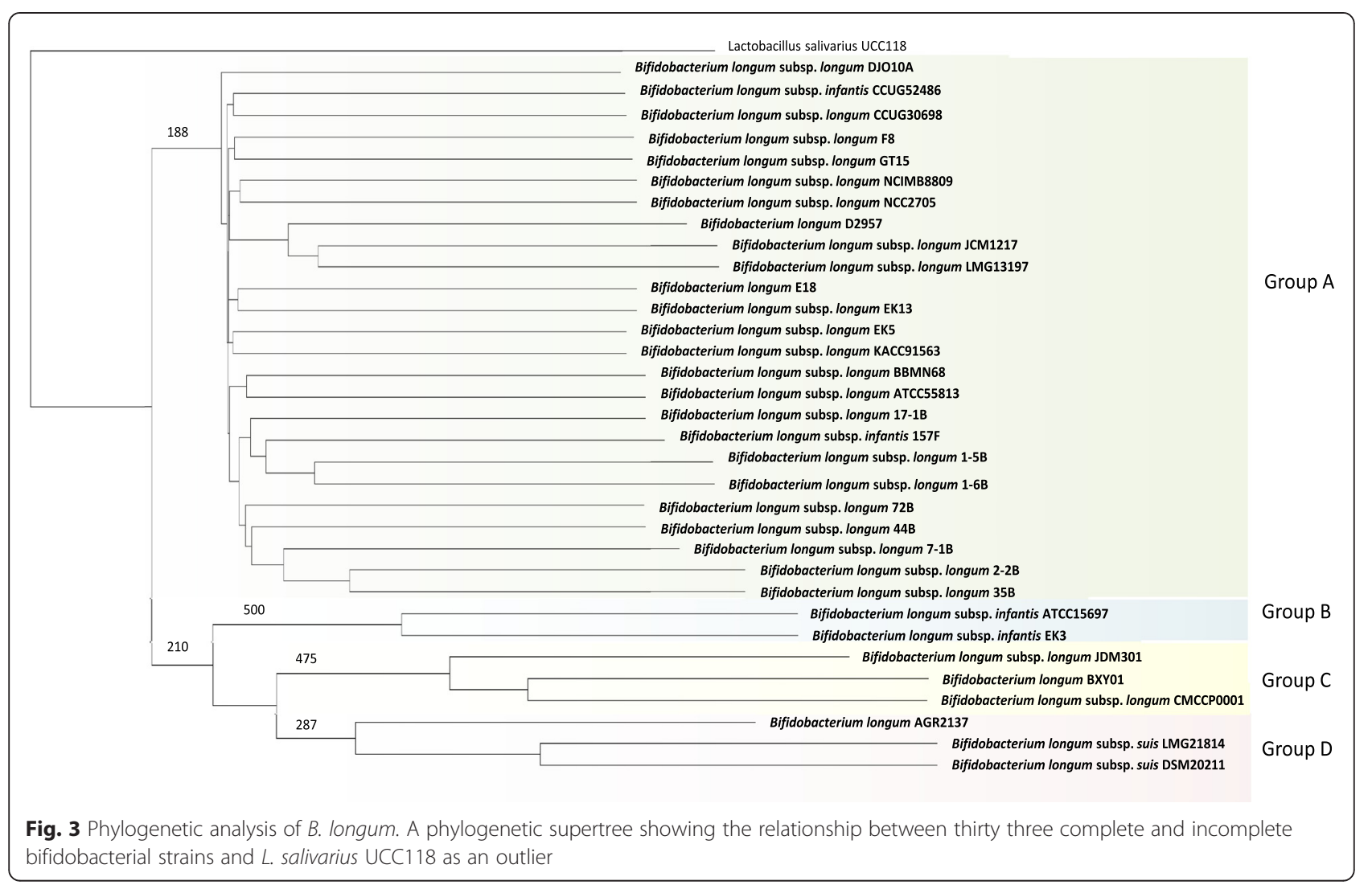

fifth subspecies which may be more prevalent amongst the Chinese population. However, validation of such a fifth subspecies and its possible geographical delineation will require further genome sequencing of additional $B$. longum isolates.

A phylogenetic approach using seven house-keeping genes has previously been used to differentiate between bifidobacterial (sub) species and it was found that this method allowed for a significant increase in the discriminatory power between taxa as compared to more traditional methods, for example $16 \mathrm{~s}$ rRNA alignments [46]. This approach was explored using several housekeeping genes and the resulting phylogenetic tree was the same as that generated from the core B. longum genome (Data not published).

\section{In silico prediction of $B$. longum metabolic capabilities}

To elucidate possible correlations between the genomic content of the analysed B. longum strains and particular phenotypic abilities, we analysed the predicted metabolic capabilities of each strain (as summarised in Table 3). Taking into account the current subspecies classification, B. longum subsp. infantis ATCC 15697 and B. longum subsp. infantis EK3 are characteristic representatives of the infantis subspecies (Additional file 3: Table S4). The genome sequences of both strains reveal that they are typical human milk oligosaccharide (HMO) utilisers and that they encode a considerably lower number of GHs that are associated with the catabolism of plant-derived polysaccharides (Table 3). This was verified phenotypically for B. longum subsp. infantis ATCC 15697, which is unable to utilise plant-derived polysaccharides and the pentose sugars arabinose and xylose, which represent the monosaccharides that are found in many plantderived polysaccharides (Fig. 4).

In silico analysis revealed that group A strains (Fig. 3) are characteristic members of the longum subspecies (Additional file 3: Table S4) and are tailored towards the metabolism of plant-derived carbohydrates (Table 3). A high variability in the number of predicted GHs, specialised in the degradation of plant polysaccharides, was observed across all analysed genomes from these Group A subspecies longum representatives (Table 3). Phenotypic analysis demonstrated the ability of $B$. longum subsp. longum NCIMB 8809 and B. longum subsp. longum CCUG 30698 to utilise various plant-derived polysaccharides and plant-associated pentose sugars (Fig. 4). As is characteristic for members of this subspecies, the strains located in group A lack the majority of genes that have previously been shown to be required for HMO utilisation (Table 3).

Both $B$. longum subsp. suis strains analysed adhere to the current classification of the suis subspecies as both strains are isolated from porcine faeces and have a 
Table 3 Presence or absence of LNB/GNB, sialic acid and HMO gene clusters in all B. longum genomes

\begin{tabular}{|c|c|c|c|c|c|c|}
\hline & $\begin{array}{l}\text { LNB/ } \\
\text { GNB }\end{array}$ & Sialic acid & HMO cluster & $\begin{array}{l}\text { Total number } \\
\text { of GHs }\end{array}$ & $\begin{array}{l}\text { GHs active against plant } \\
\text { polysaccharide }\end{array}$ & $\begin{array}{l}\text { Arabinose/Xylose } \\
\text { metabolism }\end{array}$ \\
\hline $\begin{array}{l}\text { B. longum subsp. infantis } \\
\text { ATCC } 15697\end{array}$ & + & + & + & 25 & 2 & - \\
\hline B. longum subsp. infantis EK3 & + & + & + & 26 & 2 & $+(X y l o s e ~ o n l y)$ \\
\hline $\begin{array}{l}\text { B. longum subsp. suis } \\
\text { LMG } 21814\end{array}$ & + & $\begin{array}{l}+ \text { (with the } \\
\text { exception of } n a n H)\end{array}$ & - & 32 & 5 & + \\
\hline $\begin{array}{l}\text { B. longum subsp. suis } \\
\text { DSM } 20211\end{array}$ & + & $\begin{array}{l}+ \text { (with the } \\
\text { exception of nanH) }\end{array}$ & - & 35 & 8 & + \\
\hline B. longum AGR2137 & + & - & - & 38 & 7 & + \\
\hline $\begin{array}{l}\text { B. longum subsp. longum } \\
72 \mathrm{~B}\end{array}$ & + & - & - & 37 & 7 & + \\
\hline B. longum subsp. longum EK5 & + & - & - & 34 & 8 & + \\
\hline B. longum subsp. longum GT15 & + & - & - & 36 & 9 & + \\
\hline B. longum subsp. longum JDM301 & + & - & $\begin{array}{l}\text { BLJ_0318 - } \\
\text { BLJ_0322 }\end{array}$ & 37 & 9 & + \\
\hline $\begin{array}{l}\text { B. longum subsp. longum } \\
\text { KACC91563 }\end{array}$ & + & - & - & 39 & 9 & + \\
\hline B. longum subsp. longum BXY01 & + & - & $\begin{array}{l}\text { BXY01_0319 - } \\
\text { BXY01_0323 }\end{array}$ & 37 & 10 & + \\
\hline $\begin{array}{l}\text { B. longum subsp. longum } \\
\text { CMCCP0001 }\end{array}$ & + & - & $\begin{array}{l}\text { CMCCP0001_1673-- } \\
\text { CMCCP0001_1677 }\end{array}$ & 38 & 10 & + \\
\hline B. longum subsp. infantis $157 \mathrm{~F}$ & + & - & - & 41 & 10 & + \\
\hline B. longum subsp. longum BBMN68 & + & - & - & 38 & 10 & + \\
\hline B. longum subsp. longum 171B & + & - & - & 43 & 11 & + \\
\hline B. longum subsp. longum E18 & + & - & - & 37 & 11 & + \\
\hline B. longum subsp. longum F8 & + & - & - & 43 & 11 & + \\
\hline $\begin{array}{l}\text { B. longum subsp. longum } \\
\text { NCIMB } 8809\end{array}$ & + & - & - & 38 & 13 & + \\
\hline B. longum subsp. longum D2957 & + & - & - & 39 & 12 & + \\
\hline B. longum subsp. longum EK13 & + & - & - & 39 & 12 & + \\
\hline B. longum subsp. longum JCM 1217 & + & - & - & 38 & 12 & + \\
\hline $\begin{array}{l}\text { B. longum subsp. longum LMG } \\
13197\end{array}$ & + & - & - & 37 & 13 & + \\
\hline B. longum subsp. longum NCC2705 & + & - & - & 33 & 13 & + \\
\hline B. longum subsp. longum 15B & + & - & - & 39 & 15 & + \\
\hline B. longum subsp. longum DJO10A & + & - & - & 45 & 16 & + \\
\hline $\begin{array}{l}\text { B. longum subsp. infantis } \\
\text { CCUG } 52486\end{array}$ & + & - & - & 43 & 17 & + \\
\hline $\begin{array}{l}\text { B. longum subsp. longum } \\
\text { ATCC } 55813\end{array}$ & + & - & - & 48 & 17 & + \\
\hline $\begin{array}{l}\text { B. longum subsp. longum CCUG } \\
30698\end{array}$ & + & - & - & 45 & 18 & + \\
\hline B. longum subsp. longum 16B & + & - & - & 50 & 19 & + \\
\hline B. longum subsp. longum 22B & + & - & - & 50 & 19 & + \\
\hline B. longum subsp. longum 35B & + & - & - & 50 & 19 & + \\
\hline B. longum subsp. longum 44B & + & - & - & 50 & 19 & + \\
\hline B. longum subsp. longum $71 \mathrm{~B}$ & + & - & - & 50 & 19 & + \\
\hline
\end{tabular}




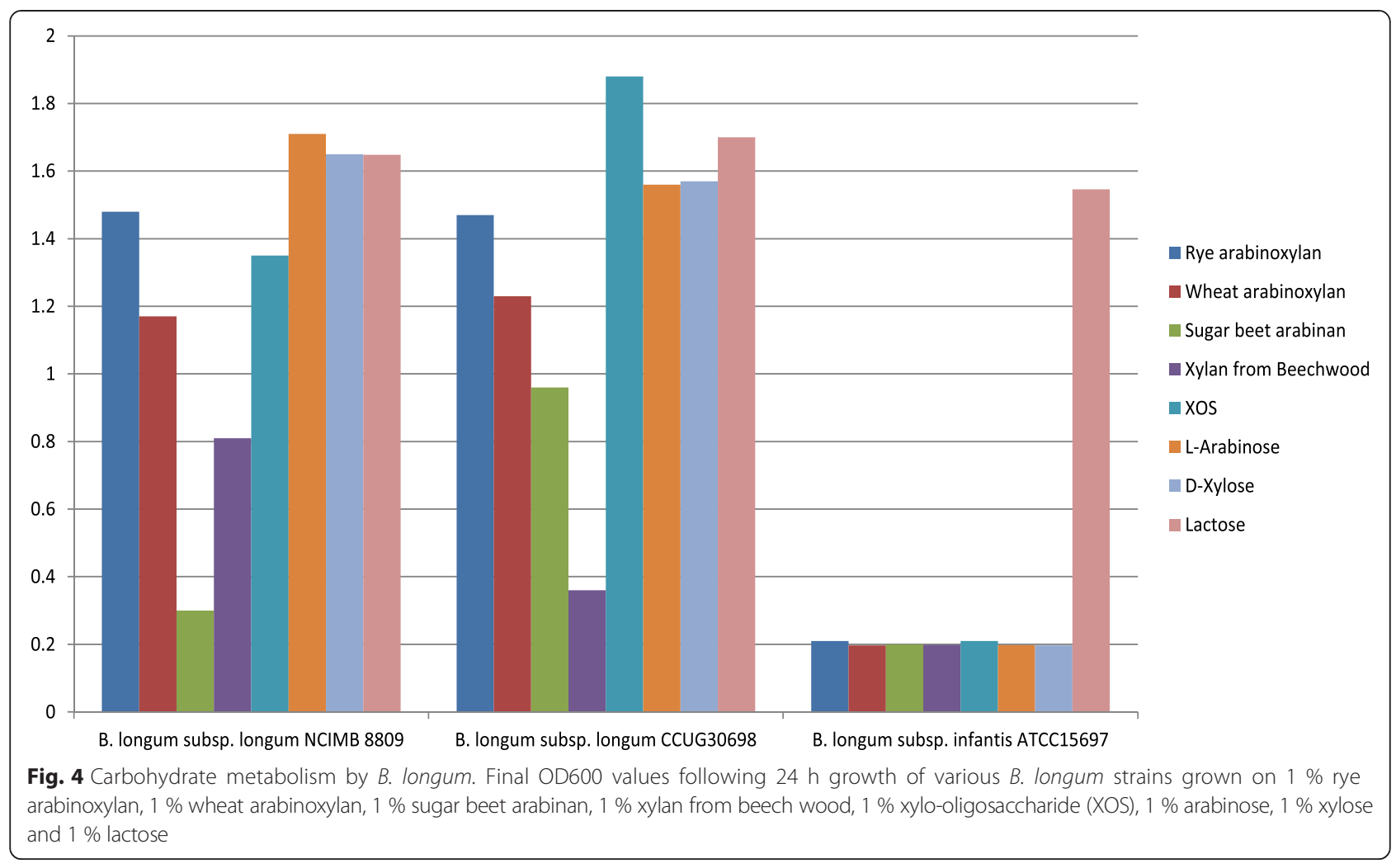

predicted ability to metabolise a range of different carbohydrates including pentoses (Fig. 3, Table 3). In addition, both strains encode a near complete sialic acid metabolism cluster, yet appear to lack the sialidase-encoding $n a n H$, presumed to be responsible for removing terminal sialic acid residues from oligosaccharides found in HMOs and/or mucin [47]. Though nanH is absent in both strains, they may still be capable of growth on sialic acid as each strain is predicted to possess the transport system necessary for the internalisation of sialic acid [47]. The third animal isolate, B. longum AGR2137, is more similar to members of the subspecies longum as this strain only harbours a partial sialic acid metabolism cluster.

Genomic data analysis of the strains $B$. longum subsp. longum BXY01, B. longum subsp. longum JDM301 and $B$. longum subsp. longum CMCCP0001 provides further evidence that these strains may represent a distinct subspecies (Fig. 3). These strains do not fit the current classification for the three B. longum subspecies as these strains are predicted to metabolise plant-derived polysaccharides, yet also appear to possess a considerable number of HMO-metabolising capabilities (Table 3 and Additional file 3: Table S4).

\section{Assessment of genetic accessibility and methylome analysis}

Firstly, to investigate how genetically accessible $B$. longum subsp. longum NCIMB 8809 and B. longum subsp. longum
CCUG 30698 are, the transformation frequency of two $E$. coli-bifidobacterial shuttle vectors, pPKCM7 and pAM5 was determined (Table 4). The approach for this involved introducing plasmid DNA that had originated from both B. longum subsp. longum strains (i.e. plasmid DNA that is protected from native R-M systems) whereas plasmid DNA isolated from E. coli EC101 was used as a control. It is worth noting that, as the plasmid DNA is isolated from two different microorganisms with different isolation protocols, there may be differences in the purity of plasmid DNA used for transformation, which could be a confounding factor. A three-log increase in transformation efficiency was observed for pAM5 and a single-log increase in transformation efficiency was observed for pPKCM7 when the respective plasmid DNA had been isolated from B. longum subsp. longum NCIMB 8809 relative to the situation when DNA isolated from E. coli had been used for the transformation (Fig. 5). Unfortunately, we were unuable to introduce either of the two plasmids in B. longum subsp. longum CCUG 30698 and therefore could not test this strain. These results indicate that B. longum subsp. longum NCIMB 8809 encodes functional R-M systems that negatively impact on the transformation efficiency of this strain when unmethylated DNA is used. B. longum subsp. longum CCUG 30698 is also likely to encode one or more potent R-M systems given its complete recalcitrance to transformation. 
Table 4 Bacterial strains and plasmids used in this study

\begin{tabular}{|c|c|c|c|}
\hline Strain/plasmid & Relevant characteristics & Additional Information & Reference or source \\
\hline \multicolumn{4}{|l|}{ E.coli strains } \\
\hline EC101 & Cloning host, rep $A^{+} \mathrm{km}^{\mathrm{r}}$ & & [51] \\
\hline EC101 pNZEM-M.blmncll & Containing M.blmNCll & Type II R-M MTase B8809_0607 & This study \\
\hline EC101 pNZEM -M.blmnclll & Containing M.blmNCIII & Type II R-M MTase B8809_0958 & This study \\
\hline EC101 pWSK29-MS.blmncl & Containing MS1.b/mNCl & $\begin{array}{l}\text { Type I R-M MTase and HsdS1 B8809_1353 } \\
\text { and B8809_1354 }\end{array}$ & This study \\
\hline EC101 pNZ44-S.blmncl & Containing $52 . b 1 \mathrm{mNCl}$ & Type I R-M HsdS2 B8809_1352 & This study \\
\hline EC101 pWSK29-MSS.blmncl & Containing MS1.blm NCl and p44+52.blm NCl & $\begin{array}{l}\text { Type I R-M MTase, HsdS1 and HsdS2 } \\
\text { B8809_1353, B8809_1354 and B8809_1352 }\end{array}$ & This study \\
\hline \multicolumn{4}{|l|}{ B.longum strains } \\
\hline NCIMB 8809 & Isolated from nursling stool & & \\
\hline CCUG 30698 & Isolated from adult intestine & & \\
\hline NCIMB 8809-ArfB & $\begin{array}{l}\text { pORI19-tetMod-ArfB insertion mutant of } \\
\text { NCIMB } 8809\end{array}$ & $\begin{array}{l}579 \text { bp internal fragment of } \operatorname{arfB} \text { and } \\
\text { tetMod gene }\end{array}$ & This study \\
\hline \multicolumn{4}{|l|}{ Plasmids } \\
\hline pAM5 & $\mathrm{pBC1}-\mathrm{puC19-Tc^{r }}$ & & [70] \\
\hline pPKCM7 & pblueCm harbouring rep pCIBA089 & & [71] \\
\hline pNZ44 & $\mathrm{Cm}^{r}$, expression vector & & [72] \\
\hline pNZ44-S.blncl & pNZ44 derivative containing blncls & & This study \\
\hline pWSK29 & & & [73] \\
\hline pNZ8048-Em & Emr; nisin-inducible translational fusion vector & & [74] \\
\hline pNZEM -M.blmncll & pNZEM derivative containing M.blmNCll & Type II R-M MTase B8809_0607 & This study \\
\hline pNZEM -M.blmnclll & pNZEM derivative containing M.blmNCIII & Type II R-M MTase B8809_0958 & This study \\
\hline pWSK29-MS.blmncl & pWSK29 derivative containing MS1.blm NCl & $\begin{array}{l}\text { Type I R-M MTase and HsdS1 B8809_1353 } \\
\text { and B8809_1354 }\end{array}$ & This study \\
\hline pNZ44-S.blmncl & pNZ44 derivative containing $52 . b 1 \mathrm{mNCl}$ & Type I R-M HsdS2 B8809_1352 & This study \\
\hline pWSK29-MSS.blmncl & $\begin{array}{l}\text { pWSK29 derivative containing MS1.blmNCl } \\
\text { and } p 44+52 . b / \mathrm{mNCl}\end{array}$ & $\begin{array}{l}\text { Type I R-M MTase, HsdS1 and HsdS2 } \\
\text { B8809_1353, B8809_1354 and B8809_1352 }\end{array}$ & This study \\
\hline pORI19 & Emr, repA-, ori+, cloning vector & & [51] \\
\hline pORI19-tet-ArfB & $\begin{array}{l}\text { Internal } 579 \text { bp fragment of arfB and tetW } \\
\text { cloned into pORI19 }\end{array}$ & & This study \\
\hline
\end{tabular}

Genome analysis of B. longum subsp. longum NCIMB 8809 and B. longum subsp. longum CCUG 30698 clearly indicates that each strain specifies one or more complete R-M systems and/or orphan MTases (Table 5). Methylome analysis was also employed (See Materials and Methods) which allowed for the identification of a number of distinct methylation recognition sequences (Table 5). It should be noted that methylated cytosines are not faithfully detected and require specific detection methods [48, 49].

This analysis revealed the recognition motif $5^{\prime}$ $\mathrm{G}^{6 \mathrm{~m}} \mathrm{ATNNNNNTGCC-3}$ ' in B. longum subsp. longum NCIMB 8809, and the two recognition motifs $5^{\prime}$ ACCNNNNNRTTG-3' and 5' -GCANNNNNNNNTGC-3' in B. longum subsp. longum CCUG 30698 (Table 5). These motifs are reminiscent of type I R-M recognition sequences [16] (Table 5). Both strains are indeed predicted to encode a single and apparently complete type I R-M system encompassing a single HsdM subunit, multiple HsdS subunits, and a one HsdR subunit, responsible for modification, restriction and sequence recognition specificity (Table 5, Additional file 4: Figure S2). It has been observed that in other organisms possessing multiple HsdS subunitencoding genes, novel specificities can be generated by recombination events among such $h s d S$ sequences [50].

B. longum subsp. longum NCIMB 8809 is also predicted to encode one complete type II R-M system and one orphan type II R-M N-4 cytosine MTase (Table 5). Based on REBASE interrogation, the REase associated with the complete type II R-M system is predicted to recognize the DNA sequence, 5'-CCWGG-3' (Table 5). However, methylome analysis only identified one type II recognition motif, 5'-TCGG ${ }^{\mathrm{m} 4} \mathrm{CCGA}-3^{\prime}$, which we predict to be associated with the presumed orphan type II 


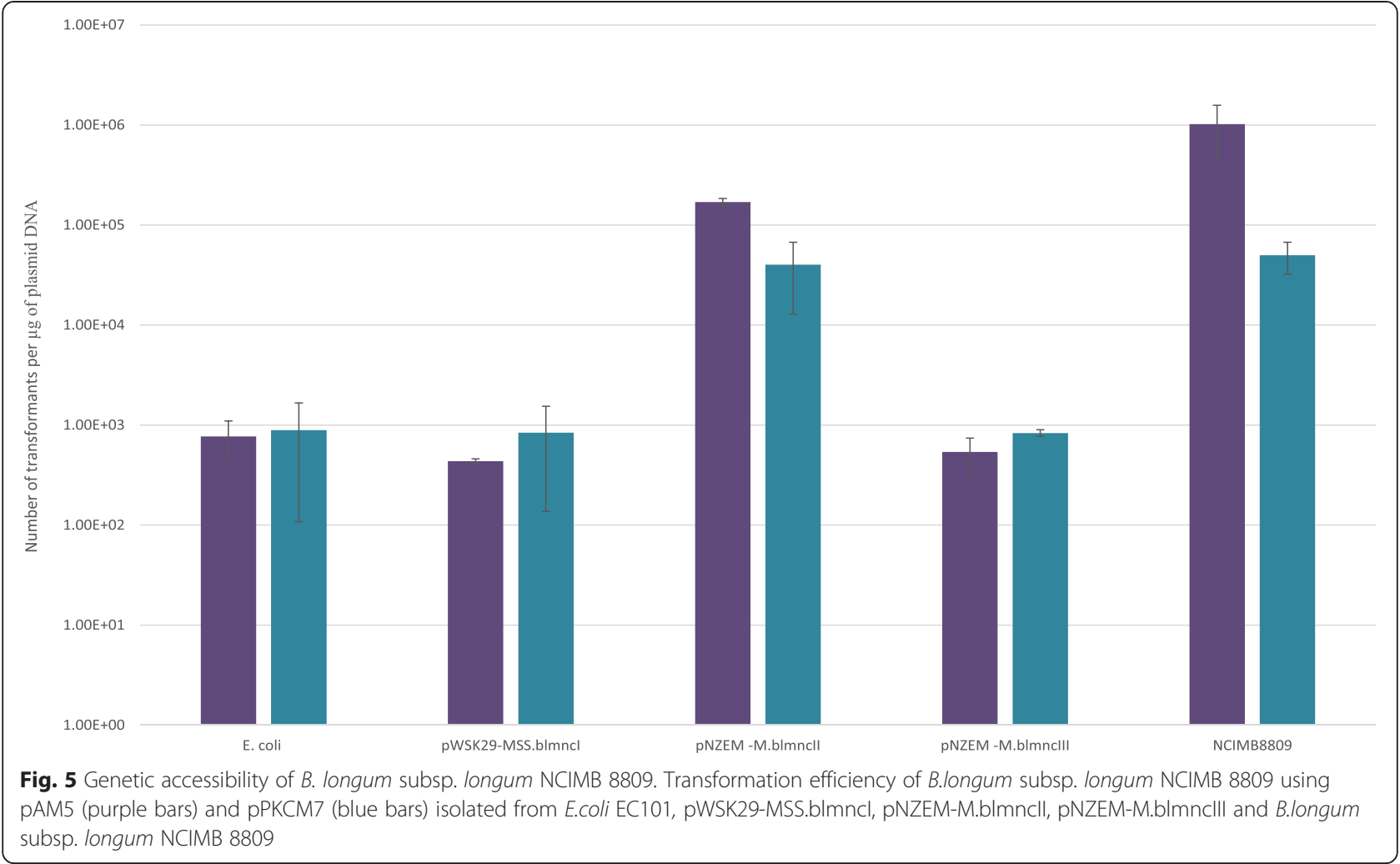

MTase (Table 5). B. longum subsp. longum CCUG 30698 is also predicted to encode a type II R-M system that is represented by an REse and N6-adenine MTase. Based on comparisons to REBASE and methylome analysis, we predict that this type II R-M system recognises the motif, 5'-CTGC ${ }^{\mathrm{m} 6} \mathrm{AG}-3$ ' (Table 5). REBASE searches also revealed the presence of what appears to be two orphaned type II MTases and an incomplete type III R-M system (Table 5). Finally, a potential type IIG R-M system was identified; based on the REBASE searches and methylome data we predict that this $\mathrm{R}-\mathrm{M}$ is associated with the recognition motif $5^{\prime}$ - $\mathrm{CGGG}^{\mathrm{m} 6}$ AG-3' (Table 5).

\section{Individual effects of $B$. longum R-M systems on transformation efficiency}

Based on the methylome data obtained and comparative analysis performed on the identified R-M systems, we wanted to verify that both $B$. longum strains encode MTases that protect the respective genomic DNA. This could only be tested for those R-M systems that are predicted to recognise the motifs $5^{\prime}$-CCWGG-3' and 5'CTGCAG-3' as commercial restriction enzymes that recognise these particular motifs are available. As expected the genomic DNA from B. longum subsp. longum NCIMB 8809 was protected from restriction with EcoRII (which cuts 5'-CCWGG-3' sequences), while it was digested by PstI (which targets 5'-CTGCAG-3' sequences). Conversely and as expected, genomic DNA from B. longum subsp. longum CCUG 30698 was protected from restriction with PstI, while it was cut by EcoRII (Additional file 5: Figure S3).

The type II R-M systems of B. longum subsp. longum NCIMB 8809 encompass the MTase-encoding genes blmNCII and blmNCIII, while the type I R-M system encompasses the HsdM-encoding gene M.blmNCI, and the (partial) HsdS-encoding genes S1.blmNCI, S2.blmNCI and S3.blmNCI. The effect of each individual R-M system on the transformation efficiency of B. longum subsp. longum NCIMB 8809 was investigated, whereas we did not pursue this for $B$. longum subsp. longum CCUG 30698 as this strain was non-transformable (see above). For this purpose, each gene encoding an MTase and specificity subunit (in the case of the type I system) were cloned in E. coli EC101 and with the exception of S2.blmNCI, all genes were cloned together with their presumed native promoter-containing region (see Materials and Methods).

Plasmids pAM5 and pPKCM7 were introduced into $E$. coli EC101 derivatives that harboured plasmids pWSK29MSS.blmncI or pNZEM-M.blmncII. Plasmid preparations of the resulting pAM5 and pPKCM7-containing E. coli EC101-derivative strains were used for $B$. longum subsp. longum NCIMB 8809 transformation. As positive controls pAM5 and pPKCM7 plasmid DNA was isolated from $B$. longum subsp. longum NCIMB 8809 (i.e. pAM5 and pPKCM7 methylated by and thus protected against from 
Table 5 R-M systems of B. longum subsp. longum NCIMB 8809 and B. longum subsp. longum CCUG 30698

\begin{tabular}{|c|c|c|c|c|c|c|}
\hline Strain & $\begin{array}{l}\text { R-M } \\
\text { type }\end{array}$ & Locus tag & $\begin{array}{l}\text { Predicted } \\
\text { function }\end{array}$ & REBASE comparison & Predicted associated motif & $\begin{array}{l}\text { nSites on } \\
\text { pAM5 }\end{array}$ \\
\hline \multirow[t]{9}{*}{$\begin{array}{l}\text { B. longum subsp. longum } \\
\text { NCIMB } 8809\end{array}$} & \multirow[t]{6}{*}{ I } & $\begin{array}{l}\text { B8809_1350 } \\
\text { (S3.blmNCI) }\end{array}$ & HsdS subunit & $\begin{array}{l}100 \% \text { with } \\
\text { Bbr12LORF1341 }\end{array}$ & \multirow[t]{2}{*}{ 5'-GATNNNNNTGCC-3'/ } & \multirow[t]{6}{*}{1} \\
\hline & & B8809_1351 & Recombinase & - & & \\
\hline & & B8809_1352(S2.blmNCl) & HsdS subunit & $\begin{array}{l}100 \% \text { with } \\
\text { Bbr12LORF1345 }\end{array}$ & \multirow[t]{4}{*}{ 3'CTANNNNNACGG-5** } & \\
\hline & & B8809_1353(S1.blmNCl) & HsdS subunit & $\begin{array}{l}100 \% \text { with } \\
\text { Bbr12LORF1345 }\end{array}$ & & \\
\hline & & B8809_1354(M.blmNCl) & MTase & $\begin{array}{l}100 \% \text { with } \\
\text { BloF8ORF1960P }\end{array}$ & & \\
\hline & & B8809_1355 (R.blmNCl) & RTase & $\begin{array}{l}100 \% \text { with } \\
\text { BloF8ORF1960P }\end{array}$ & & \\
\hline & \multirow[t]{2}{*}{$\|$} & $\begin{array}{l}\text { B8809_0606 } \\
\text { (R.blmNCII) }\end{array}$ & RTase & $\begin{array}{l}90 \% \text { with } \\
\text { Blo68ORF557P }\end{array}$ & \multirow[t]{2}{*}{$5^{\prime}-$ CCWGG - 3' } & \multirow[t]{2}{*}{13} \\
\hline & & B8809_0607(M.blmNCII) & MTase & $\begin{array}{l}82 \% \text { with } \\
\text { Blo68ORF556P, N5-C }\end{array}$ & & \\
\hline & $\|$ & $\begin{array}{l}\text { B8809_0959 } \\
\text { (M.blmNCIII) }\end{array}$ & MTase & $\begin{array}{l}67 \% \text { with } \\
\text { Bde27679ORF2230P, N4- } \\
\text { C }\end{array}$ & $5^{\prime}-$ TCGGCCGA - 3'* & 0 \\
\hline \multirow[t]{13}{*}{$\begin{array}{l}\text { B. longum subsp. longum } \\
\text { CCUG } 30698\end{array}$} & \multirow[t]{7}{*}{ । } & BBL306_1749 & HsdS subunit & $\begin{array}{l}42 \% \text { with } \\
\text { Cty } 7086 \text { ORFEP }\end{array}$ & \multirow[t]{4}{*}{$\begin{array}{l}5^{\prime} \text {-ACCNNNNNRTTG- 3'/ } 3^{\prime}- \\
\text { TGGNNNNNYAAC- } 5^{\prime *}\end{array}$} & \multirow[t]{4}{*}{2} \\
\hline & & BBL306_1750 & Recombinase & - & & \\
\hline & & BBL306_1751 & HsdS subunit & $\begin{array}{l}44 \% \text { with } \\
\text { BkaPV202ORF3675 }\end{array}$ & & \\
\hline & & BBL306_1752 & $\begin{array}{l}\text { Hypothetical } \\
\text { protein }\end{array}$ & - & & \\
\hline & & BBL306_1753 & MTase & $\begin{array}{l}98 \% \text { with } \\
\text { Blo44BORF2305P }\end{array}$ & \multirow[t]{3}{*}{ 5'-GCANNNNNNNNTGC-3'* } & \multirow[t]{3}{*}{4} \\
\hline & & BBL306_1754 & HsdS subunit & $\begin{array}{l}67 \% \text { with } \\
\text { BloDORF1956P }\end{array}$ & & \\
\hline & & BBL306_1756 & RTase & $\begin{array}{l}100 \% \text { with } \\
\text { BloDORF1959P }\end{array}$ & & \\
\hline & \multirow[t]{2}{*}{$\|$} & BBL306_0230 & RTase & $\begin{array}{l}100 \% \text { with } \\
\text { BloAORF289P }\end{array}$ & \multirow[t]{2}{*}{$5^{\prime}-$ CTGCAG $-3^{\prime *}$} & \multirow[t]{2}{*}{1} \\
\hline & & BBL306_0231 & MTase & $68 \%$ with BbrUIII, N6-A & & \\
\hline & $\|$ & BBL306_0271 & MTase & $\begin{array}{l}100 \% \text { with } \\
\text { M.Blo7210ORFAP, N6-A }\end{array}$ & Unknown & Unknown \\
\hline & $\|$ & BBL306_1693 & MTase & $\begin{array}{l}10 \% \text { Pae9BRORFBP,N4- } \\
\text { C/N6-A }\end{array}$ & Unknown & Unknown \\
\hline & $\| G$ & BBL306_1028 & RTase/MTase & $\begin{array}{l}98 \% \text { with } \\
\text { Blo1217ORF992P, N6-A }\end{array}$ & $5^{\prime}-$ CGGGAG - 3'* & 5 \\
\hline & III & BBL306_0910 & MTase & $\begin{array}{l}45 \% \text { with } \\
\text { Bwa914ORF4853, N6-A }\end{array}$ & Unknown & Unknown \\
\hline
\end{tabular}

Methylated bases are indicated in bold red text. The " "*" indicates sites that were identified by methylome analysis

the native R-M systems), whereas pAM5 and pPKCM7 plasmid DNA isolated from E. coli EC101 acted as the negative control (i.e. unprotected pAM5 and pPKCM7).

The highest observed transformation efficiency was achieved when plasmid DNA was isolated from the $E$. coli EC101 derivative harbouring plasmid pNZEMM.blmncII (Fig. 5). This observation is not surprising as the motif associated with this MTase has the highest number of occurrences on pAM5 compared to the other recognition motifs identified (Table 5). Our data indicate that the type I R-M system present in B. longum subsp. longum NCIMB 8809 does not have a negative effect on transformation efficiency. This result may be due to the low number of recognition motifs present on pAM5 that are associated with this type I R-M system or to the possibility that this system is inactive (Table 5). The 
transformation efficiency for the positive control (pAM5 plasmid DNA isolated from B. longum subsp. longum NCIMB 8809) is still 10-fold higher than that achieved for plasmid DNA isolated from the E. coli EC101 derivative harbouring plasmid pNZEM-M.blmncII, which suggests that the latter plasmid is not fully protected against the endogenous restriction activity of NCIMB 8809. Furthermore, the second type II R-M system present in $B$. longum subsp. longum NCIMB 8809 does not negatively impact on the transformation efficiency of this strain and may be due to the fact that this R-M system is nonfunctional.

\section{Disruption of the arfB in B. longum subsp. longum NCIMB 8809}

The $\operatorname{arf} B$ gene was selected as a mutational target in order to determine whether methylation of the non-replicating plasmid by the B. longum subsp. longum NCIMB 8809 MTase M.blmncII would increase transformation efficiency to allow for site-specific homologous recombination. The $\operatorname{arf} B$ gene encodes a putative $\alpha$-L-arabinofuranosidase (Araf) predicted to be involved in the degradation of the plant-derived carbohydrate arabinoxylan through the hydrolysis of $\alpha$-L-arabinofuranosyl residues from the xylan backbone of arabinoxylan. The DNA fragment of $579 \mathrm{bp}$, representing an internal fragment of the gene $\operatorname{arfB}$, was cloned in pORI19 to generate construct pORI19-ArfB (see Materials and Methods). The pORI19 derivative, pORI19$A r f B$, was provided with the tetracycline marker, tet $W_{\text {Mod, }}$, resulting in the plasmid, pORI19-tetMod-ArfB. We used a synthetic tet $W_{\text {Mod }}$ gene, which is free of EcoRII restriction sites compared to the original tet $W$ sequence which contains a total of seven EcoRII sites (we noticed that plasmid DNA isolated from E. coli pNZEM-M.blmncII was not fully methylated and therefore was still vulnerable to (partial) restriction (Additional file 6: Figure S4, panel a)). There are two EcoRII sites present in the selected internal arfB fragment, whereas the non-replicative pORI19 plasmid contains just a single EcoRII site (and therefore pORI19tetMod-ArfB contains three EcoRII sites). This pORI19 derivative is unable to replicate in B. longum subsp. longum NCIMB 8809 as it lacks a functional replication protein [51]. In order to methylate the constructed plasmid pORI19-tetMod-ArfB, this plasmid was introduced into $E$. coli pNZEM-M.blmncII, expressing the type II MTase from B. longum subsp. longum NCIMB 8809. Restriction analysis revealed that plasmid pORI19-tetMod-ArfB (when isolated from E. coli pNZEM-M.blmncII) was protected from restriction with EcoRII (Additional file 6: Figure S4, panel b). Restriction analysis also revealed that the un-methylated pORI19-tetMod-ArfB plasmid (i.e. not introduced into $E$. coli pNZEM-M.blmncII) was subject to restriction by EcoRII (data not shown).

Methylated pORI19-tetMod-ArfB was then introduced into B. longum subsp. longum NCIMB 8809 via electroporation. Several $\mathrm{Tet}^{\mathrm{r}}$ transformants were obtained and analysed by colony PCR, which indeed verified that the individual pORI19-derivative had integrated at the expected chromosomal location. To assess the phenotypic effect of the gene disruption created in $\operatorname{arfB}$, a selected mutant, designated $B$. longum subsp. longum NCIMB 8809-ArfB, was analysed for its ability to grow on mMRS supplemented with arabinoxylan as the sole carbon source. In contrast to (wild type) B. longum subsp. longum NCIMB 8809, the arfB-mutant exhibited a complete inability to grow on both rye and wheat arabinoxylan (though retained, as expected, the ability to grow on lactose as a sole carbon source) (Fig. 6).

This result shows that $\operatorname{arfB}$ is required for growth of B. longum subsp. longum NCIMB 8809 on arabinoxylan. Based on the predicted function of ArfB we speculate that this enzyme cleaves the arabinose substitutions

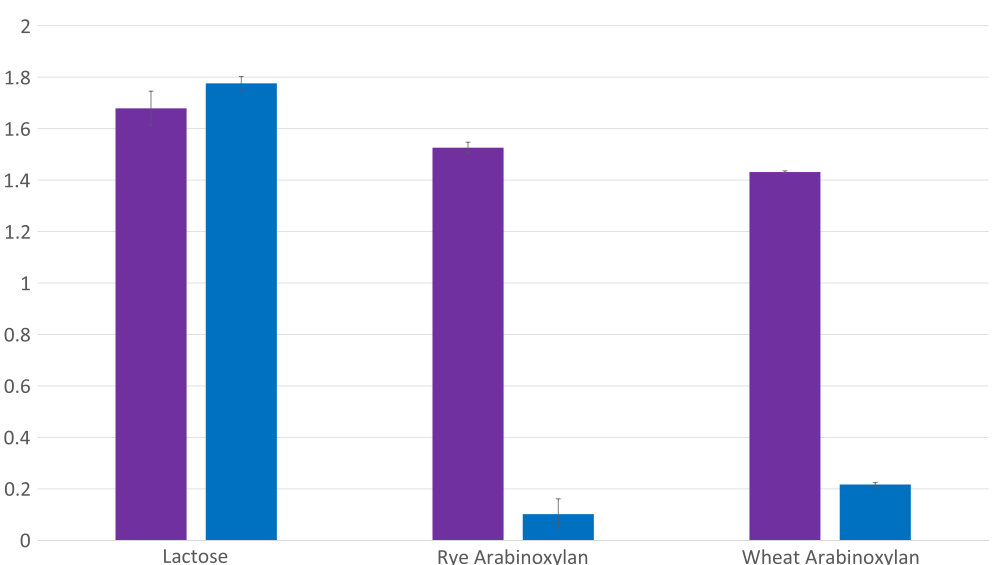

Fig. 6 Phenotypic analysis of the B. longum subsp. longum NCIMB 8809 insertion mutant strain. Growth profile analysis of B.longum subsp. longum NCIMB 8809 (purple) and B.longum subsp. longum NCIMB 8809-ArfB (blue) in modified Rogosa broth supplemented with lactose, rye arabinoxylan or wheat arabinoxylan 
from arabinoxylan and that the released arabinose moieties are then internalized and metabolised.

\section{Conclusions}

Comparative genomics of bifidobacteria may be very helpful in order to expand our understanding of (bifido)bacterial-host interactions, whereby they may elicit health benefits, and of the ability for bifidobacteria to adapt and compete in the intestinal environment. The full genome sequencing of two human $B$. longum isolates and the subsequent analysis of all complete and incomplete $B$. longum genomes allowed for the description of the pan- and core-genome for the B. longum species. This analysis revealed that the B. longum pan-genome is essentially closed and that the genomes included in this analysis are likely to be sufficient in describing the genetics and derived biology of this bifidobacterial species.

It is evident from the determined genome sequences, which are comparable to other fully sequenced $B$. longum genomes, that $B$. longum subsp. longum NCIMB 8809 and $B$. longum subsp. longum CCUG 30698 possess various genetic adaptations and associated ecological fitness to suit life in the GIT. In light of the current B. longum subspecies classification, the phenotypic, phylogenetic and in silico analysis presented here has provided evidence for the existence of a novel phylogenetic type, which may represent a fifth subspecies. To determine whether further subclassification is desirable and can be justified, additional sequencing of $B$. longum isolates genomes is required. We also propose an amendment to the description of the subspecies infantis in that members may have capabilities to utilise carbohydrates other than HMOs. We also provide evidence that indicates that $B$. longum subsp. infantis $157 \mathrm{~F}$ and B. longum subsp. infantis CCUG 52486 should be assigned to the longum subspecies [52]. Finally, comparative analysis of B. longum highlights the versatility of this species and its ability to move with us from infancy to adulthood.

The genetic inaccessibility of bifidobacteria hinders the exploration of the molecular mechanisms that are responsible for its acclaimed probiotic activities. Improvement of transformation efficiency has previously been achieved by overcoming R-M systems $[14,15]$. The negative impact of R-M systems on transformation efficiency of bifidobacteria has been demonstrated in $B$. breve UCC2003 [15], B. adolescentis ATCC 15703 [20] and B. bifidum S17 [53]. However, such an R-M barrier can be overcome by the appropriate pre-methylation of plasmid DNA [15, 20, 21, 54].

By exploiting our knowledge of the active R-M systems harboured by B. longum subsp. longum NCIMB 8809, we successfully generated a mutant of this strain via homologous recombination. The mutant strain $B$. longum subsp. longum NCIMB 8809-ArfB revealed that $\operatorname{arfB}$ plays a vital role in arabinoxylan degradation.

\section{Methods}

Bacterial strains and growth conditions

Bacterial strains and plasmids used in this study are detailed in Table 4. Bifidobacteria were routinely cultured in modified de Man, Rogosa and Sharpe (mMRS). This medium was made from first principles [55], though excluding a carbohydrate source, and then supplemented with $1 \%$ (wt/vol) lactose (unless otherwise specified) and $0.05 \%(\mathrm{wt} / \mathrm{vol})$ cysteine- $\mathrm{HCl}$. Cultures were incubated at $37{ }^{\circ} \mathrm{C}$ under anaerobic conditions which were maintained using an Anaerocult oxygen depletion system (Merck, Darmstadt, Germany) in an anaerobic chamber with an atmosphere of $5 \% \mathrm{CO}_{2}-5 \% \mathrm{H}_{2}-90 \% \mathrm{~N}_{2}$.

Escherichia coli strains were cultured in LB broth (LB) $[56,57]$ at $37{ }^{\circ} \mathrm{C}$ with agitation. Where appropriate, growth media contained chloramphenicol $\left(\mathrm{Cm} ; 10 \mu \mathrm{g} \mathrm{ml}{ }^{-1}\right.$ for $E$. coli and $2 \mu \mathrm{g} \mathrm{ml}^{-1}$ for B. longum), erythromycin (Em; $100 \mu \mathrm{g} \mathrm{ml}^{-1}$ for E. coli) ampicillin (Amp; $100 \mu \mathrm{g} \mathrm{ml}^{-1}$ for $E$. coli), tetracycline (Tet; $10 \mu \mathrm{g} \mathrm{ml}^{-1}$ for $E$. coli and $B$. longum) or kanamycin (Km; $50 \mu \mathrm{g} \mathrm{ml}^{-1}$ for E. coli).

\section{PacBio SMRT Sequencing, data assembly and methylome analysis}

Chromosomal DNA from B. longum subsp. longum NCIMB 8809 and B. longum subsp. longum CCUG 30698 was isolated as previously described [58] and purified using the PowerClean DNA Clean-Up Kit by MoBio Laboratories (Carlsbad, CA). SMRT bell library preparation was performed as previously described [59, 60]. SMRT sequencing was performed on a PacBio RS instrument (executed by GATC Biotech, Germany) and assembled using the Pacific Biosciences SMRTPortal analysis platform (version 2.1.1). Illumina sequencing was performed by the commercial sequencing service providers Macrogen (Seoul, Republic of Korea) (using a paired-end library). The Illumina sequences obtained were then assembled with the SMRTPortal output using MIRA v3.9 (http://www.chevreux.org/projects_mira.html). Remaining gaps and quality issues were resolved using Sanger sequencing of PCR products.

To identify methylated positions the Pacific Biosciences SMRTPortal analysis platform (version 1.4) was adopted, this employs an in silico kinetic reference and a $t$-test based kinetic score detection of modified base positions.

\section{General features prediction}

Prediction of putative open reading frames (ORFs) was performed using PRODIGAL prediction software (http:// prodigal.ornl.gov/) and supported by BLASTX [61] alignments. Results of Prodigal/BLASTX were combined 
manually and a preliminary identification of ORFs was performed on the basis of BLASTP [61] analysis against a non-redundant protein database provided by the National Centre for Biotechnology (http://www.ncbi.nlm.nih.gov/). Using the ORF finding outputs and associated BLASTP results, Artemis [62] was employed for visualisation and manual editing in order to verify, and, where necessary, redefine the start of every predicted coding region, or to remove or add coding regions. The assignment of protein function to predicted coding regions was performed manually. In addition, the individual members of the revised gene/protein data set were searched against the protein family (Pfam) [63] and COG [64] databases. Ribosomal RNA (rRNA) and transfer RNA (tRNA) genes were detected using RNAMMER (http://www.cbs.dtu.dk/ services/RNAmmer/) and tRNA-scanSE (http://lowelab. ucsc.edu/tRNAscan-SE/), respectively. COG category assignment [64] was performed by means of BLASTP [61] analysis against the COG database [65] for deduced proteins of all identified ORFs contained by the genomes of both $B$. longum strains that were sequenced as part of the current study, and of all publicly available B. longum strains.

The genome sequences of both $B$. longum subs. longum strains were searched for the presence of Restriction/Modification systems using a BLASTP [61] alignment function of the REBASE database (http:// rebase.neb.com/rebase/rebase.html) (cut-off e-value of 0.00001 ; and showing at least $30 \%$ similarity across $80 \%$ of the protein length).

\section{Pan-genome determination}

Where relevant, genomes were re-annotated using the same annotation method described for $B$. longum subsp. longum NCIMB 8809 and B. longum subsp. longum CCUG 30698. For the available bifidobacterial genomes (complete and incomplete) a pan-genome computation was performed using PGAP v1.2 [66], which performs this analysis according to Heaps law pan-genome model [38]; the ORF content of each genome is organised in functional gene clusters using the GF (Gene Family) method and a pan-genome profile was then built.

\section{Phylogenetic analysis and Whole genome alignments}

The computation of a phylogenetic supertree was performed based on the alignment of a set of orthologous proteins defined by the pan-genome computation. Each protein family was aligned using CLUSTAL_W v1.83 [67]. Phylogenetic trees were computed using the maximum-likelihood in PhyML v3.0 [68] and concatenated; the resulting consensus tree was computed using the Consense module from the Phylip package v3.69 using the majority rule method (http:// evolution.genetics.washington.edu/phylip.html). Whole genome comparisons of the two newly sequenced $B$. longum strains were performed against $B$. longum subsp. longum NCC2705 (AE014295). Whole genomes were compared at the nucleotide level using MUMmer software [69] at default settings.

\section{Nucleotide sequence accession numbers}

All sequences used for our analysis were retrieved from the GenBank database and are listed with associated accession numbers in Additional file 7: Table S1.

\section{DNA manipulations}

Chromosomal DNA was isolated from B. longum subsp. longum NCIMB 8809 and B. longum subsp. longum CCUG 30698 as previously described [58]. Isolation of plasmid DNA from E. coli or B. longum was achieved by using the Roche High Pure Plasmid Isolation Kit (Roche Diagnostics). For B. longum an initial lysis step was incorporated into the plasmid isolation procedure, and cells were resuspended in lysis buffer supplemented with lysozyme $\left(30 \mathrm{mg} \mathrm{ml}^{-1}\right.$ ) and incubated at $37{ }^{\circ} \mathrm{C}$ for $30 \mathrm{~min}$. The procedures for DNA manipulations were performed essentially as described by Sambrook et al. [56]. Restriction endonucleases, shrimp alkaline phosphatase, and T4 DNA ligase were obtained from Roche Diagnostics and used according to the supplier's instructions (Roche Diagnostics, Bell Lane, East Sussex, United Kingdom). The synthetic single-stranded oligonucleotide primers used in this study, detailed in Additional file 8: Table S2, were synthesized by MWG Biotech AG (Ebersberg, Germany). Standard PCRs were performed using Taq PCR mastermix (Qiagen), while high-fidelity PCR was achieved by the use of either PfuUltra II polymerase (Agilent Technology) or LongAmp Taq polymerase (New England Biolabs). PCR amplicons were purified using the Roche High Pure PCR Purification Kit (Roche Diagnostics). Electroporation of plasmid DNA into E. coli was performed as previously described [56]. The correct orientation and integrity of all constructs was verified by DNA-sequencing, performed by Eurofins MWG Biotech.

\section{Construction of $B$. longum insertion mutant strains}

An internal fragment of arfB (corresponding to locus tag B8809_1600), encompassing 579 bp and representing codons 240 through to 433 out of the 842 codons of this gene was amplified by PCR using B. longum subsp. longum NCIMB 8809 chromosomal DNA as template and the oligonucleotide primer combination arfBMfHindIII and arfBMrXbaI (Additional file 8: Table S2). The generated PCR product was ligated to pORI19, an Ori + RepA- integration plasmid [51], using HindIII and XbaI restriction sites that were 
incorporated into the primers for this partial arfBencompassing amplicon, and introduced into $E$. coli EC101 by electroporation. Recombinant E. coli EC101 derivatives containing pORI19 constructs were selected on LB agar containing Em, and supplemented with X-gal (5-bromo-4-chloro-3-indolyl-D-galactopyranoside)

$\left(40 \mu \mathrm{g} \mathrm{ml}^{-1}\right)$ and $1 \mathrm{mM}$ IPTG.

A modified tet $W$ gene, conferring resistance to tetracyclin and free of EcoRII sites, was synthesized by Eurofins Genomics, designated here as tet $W_{\text {Mod }}$ [70]. The expected genetic structure of the recombinant plasmids pORI19-arfB (pORI19 containing an internal 579 bp fragment of the $\operatorname{arf} B$ gene) was confirmed by restriction mapping prior to subcloning of tet $W_{M o d}$ as a SacI fragment into the unique SacI site present on pORI19-arfB. The orientation of the tetracycline resistance gene in each of the resulting plasmids pORI19-tetMod-arfB, was determined by restriction analysis. Plasmid pORI19-tetMod-arfB was introduced into $E$. coli EC101 pNZEM-M.blncbII (see below, Table 4) (transformants were selected based on Tet resistance) in order to methylate the plasmid constructs before introduction into B. longum subsp. longum NCIMB 8809. A plasmid preparation of methylated pORI19tetMod-arfB was introduced into B. longum subsp. longum NCIMB 8809 by electroporation with subsequent selection on RCA plates supplemented with tetracycline [54].

Insertion mutants resulting from site-specific homologous recombination were initially confirmed by colony PCR targeting the tetracycline resistance genes tet $W_{\text {Mod }}$, followed by a second confirmatory PCR adopting tet $W_{\text {Mod }}$-based primer, either forward or reverse depending on the orientation of tet $W_{\text {Mod }}$, in combination with a primer specific for the targeted $\operatorname{arfB}$ gene to confirm integration at the expected chromosomal position (Additional file 8: Table S2). The verified mutant carrying a chromosomal insertion in $\operatorname{arf} B$ was designated as $B$. longum subsp. longum NCIMB 8809-ArfB.

\section{Transformation of $B$. longum subsp. longum NCIMB 8809}

Transformation of $B$. longum was achieved essentially according to a previously published protocol [54]. This protocol was optimised for B. longum subsp. longum NCIMB 8809 in order to achieve a transformation efficiency high enough to generate mutants via homologous recombination. The original protocol instructs that cultures are grown in MRS broth, a glucose-based medium. For the optimised protocol $B$. longum subsp. longum NCIMB 8809 was inoculated in MRS medium supplemented with $1 \%$ lactose. Furthermore, the highest transformation efficiency for $B$. longum subsp. longum NCIMB 8809 was achieved when cells were harvested once they reached an $\mathrm{OD}_{600}$ of $0.6-0.8$ (as opposed to an $\mathrm{OD}_{600}$ of 0.3 to 0.4 prescribed for the original protocol). For each transformation experiment a plasmid quantity of $400 \mathrm{ng}$ was used (isolated from either the relevant $B$. longum strain or E. coli EC101) employing electroporation of $B$. longum subsp. longum NCIMB 8809. Transformants were selected on RCA supplemented with either chlorampheni$\mathrm{col}(\mathrm{Cm})$ for plasmid pPKCM7 or tetracycline (Tet) in the case of pAM5, and enumerated following a $48 \mathrm{~h}$ incubation at $37^{\circ} \mathrm{C}$ under anaerobic conditions.

\section{Cloning of methylase-encoding genes in E. coli}

DNA fragments encompassing the genes M.blmNCII (corresponding to locus tag B8809_0607), M.blmNCIII (corresponding to locus tag B8809_0959), M.blmNCI and S1.blmNCI (corresponding to locus tags B8809_1354 and B8809_1353, respectively), and S2.blmNCI (corresponding to locus tag B8809_1352) were generated by PCR amplification from chromosomal DNA of B. longum subsp. longum NCIMB 8809 using PfuUltra II polymerase and the primer combinations found in Additional file 8: Table S2. The generated PCR amplicons were digested with restriction enzymes that correspond to the sites incorporated into the $5^{\prime}$ end of forward and reverse primers (Additional file 8: Table S2).

The digested PCR amplicons M.blmNCII and M.blmNCIII were ligated into similarly digested pNZ8048-Em. The digested PCR amplicons M.blmNCI and S1.blmNCI were ligated into similarly digested pWSK29, whereas the digested fragment encompassing S2.blmNCI was ligated into similarly digested pNZ44. The resulting ligations were introduced into $E$. coli EC101 (Table 4) by electrotransformation, and transformants were selected based on kanamycin and either ampicillin (pWSK29-based), erythromycin (pNZ8048Em-based) or chloramphenicol resistance (pNZ44-based). The plasmid content of twenty transformants was screened by restriction analysis and on average eight positive clones were identified. The integrity of five of the positively identified clones was verified by sequencing. The plasmids were designated as pNZEMM.blmncII, pNZEM-M.blmncIII, pWSK29-MS.blmncI and pNZ44-S.blmncI (see Table 4 for details). Finally, to construct plasmid pWSK29-MSS.blmncI the DNA fragment encompassing the $\mathrm{p} 44$ promoter sequence and S2.blmNCI was generated by PCR amplification from the plasmid pNZ44-S.blmncI using LongAmp Taq polymerase and primer combination found in Additional file 8: Table S2. The digested PCR amplicon was ligated to similarly digested pWSK29-MS.blmncI and introduced into E. coli EC101 (Table 4) by electrotransformation, and transformants were selected based on kanamycin and ampicillin resistance. The plasmid content of 
twenty $\mathrm{Kan}^{\mathrm{r}}$ and $\mathrm{Amp}^{\mathrm{r}}$ transformants was assessed by restriction analysis resulting in the identification of sixteen positive clones. The integrity of five of the positively identified clones was verified by sequencing.

\section{Additional files}

Additional file 1: Figure S1. Whole genome alignments. Dotplot comparison based on the genomics sequence alignments of $B$. longum subsp. longum NCC2705 to B. longum subsp. longum NCIMB 8809 and $B$. longum subsp. longum CCUG 30698. (PDF $240 \mathrm{~kb}$ )

Additional file 2: Table S3. B. longum subsp. longum NCIMB 8809 episome. An .xls document listing the predicted protein function of episomal ORFs located within the genome sequence of $B$. longum subsp. longum NCIMB 8809. (XLSX 9 kb)

Additional file 3: Table S4. Summary of the B. longum classification. An .xls document describing the classification of the three $B$. longum subspecies, B. longum subsp. longum, $B$. longum subsp. infantis and $B$. longum subsp. suis. (XLSX $12 \mathrm{~kb}$ )

Additional file 4: Figure S2. Type I R-M systems of $B$. longum. A schematic representing the type I R-M system present in B.longum subsp. longum NCIMB 8809, B.breve 12 L, B.longum subsp. longum JCM1217, B.longum subsp. longum F8 and B.longum subsp. longum CCUG 30698. Each arrow represents an ORF. The predicted protein function is indicated as Restriction (Green), Modification (Red), Specificity (Blue), Recombinase (Grey), Hypothetical (White) and Transcriptional regulator (Orange). Target recognition domains (TRDs) are indicated as orange boxes. The percentage amino acid identity is indicated as compared to the B.longum subsp. longum NCIMB 8809 type I R-M. (PDF 11 kb)

Additional file 5: Figure $\mathbf{S 3}$. Restriction analysis of $B$. longum genomic DNA. Restriction analysis of genomic DNA from $B$. longum subsp. longum NCIMB 8809 and B. longum subsp. longum CCUG 30698. Lane 1, molecular weight marker (Bioline). Lane 2: Unrestricted total DNA from B. longum subsp. longum NCIMB 8809, Lane 3 and 4 total B. longum subsp. longum NCIMB 8809 DNA restricted with lane 3 EcoRll and lane 4 Pstl. Lane 5: Unrestricted total DNA from B. longum subsp. longum CCUG 30698, Lane 6 and 7 total B. longum subsp. longum CCUG 30698 DNA restricted with lane 6 EcoRll and lane 7 Pstl. (PDF $93 \mathrm{~kb}$ )

Additional file 6: Figure S4. Restriction analysis of plasmid DNA. a) Lane 1: molecular weight marker (Bioline). Lane 2 Unrestricted pAM5 plasmid DNA isolated from E. coli pNZEM-M.blmncll. Lane 3 pAM5 plasmid DNA isolated from E. coli pNZEM-M.blmncll and restricted with EcoRII. b) Lane 1: molecular weight marker (Bioline). Lane 2 unrestricted plasmid DNA pORI19-tetMod-ArfB isolated from E. coli pNZEM-M.blmncll. Lane 3 pORI19-tetMod-ArrB plasmid DNA isolated from E. coli pNZEM-M.blmncll and restricted with EcoRll. (PDF $102 \mathrm{~kb}$ )

Additional file 7: Table S1. List of Bifidobacterium longum representatives. An .xls document with a list of all $B$. longum representatives that were used in the comparative analysis. (XLSX $13 \mathrm{~kb}$ )

Additional file 8: Table S2. Primers used in this study. An .xls document with a list of primer used in this study and sequences of restriction sites are indicated in bold text. (XLSX $11 \mathrm{~kb})$

\section{Abbreviations}

GIT: gastrointestinal tract; R-M system: restriction modification system; ORF: open reading frame; GF: gene family; COG: cluster of orthologous groups; PEP-PTS: phosphoenolpyruvate-phosphotransferase system; GH: glycosyl hydrolase; sEPS: surface exopolysaccharide; GT: glycosyl transferase; HMO: human milk oligosaccharide; Araf: arabinofuranosidase.

\section{Competing interests}

The authors declare that they have no competing interests.

\section{Authors' contributions}

AOC participated in the design and coordination of the study, performed genome assembly, genome annotation, methylome analysis, data analysis, performed experiments and insertion mutagenesis, analysed data and drafted the manuscript. FB participated in the design and coordination of the study, assisted in genome assembly, genome annotation, methylome analysis, data analysis and helped draft the manuscript. MOM participated in the design and coordination of the study, assisted in methylome analysis, experiments and insertion mutagenesis, data analysis and helped draft the manuscript. DvS conceived this study, participated in the design and coordination of the study, analysed data and helped to draft the manuscript.

\section{Acknowledgements}

This publication has emanated from research conducted within the APC Microbiome Institute with the financial support of Science Foundation Ireland (SFI) (Grant No. SFI/12/RC/2273). In addition, AOC was supported by an enterprise partnership scheme of the Irish Research Council, while MOM is a recipient of a HRB postdoctoral fellowship (Grant No. PDTM/20011/9).

Received: 21 July 2015 Accepted: 30 September 2015

Published online: 21 October 2015

\section{References}

1. Ventura M, Turroni F, Motherway MO, MacSharry J, van Sinderen D. Host-microbe interactions that facilitate gut colonization by commensal bifidobacteria. Trends Microbiol. 2012;20(10):467-76.

2. Ventura M, Canchaya C, Fitzgerald GF, Gupta RS, van Sinderen D. Genomics as a means to understand bacterial phylogeny and ecological adaptation: the case of bifidobacteria. Antonie Van Leeuwenhoek. 2007;91(4):351-72.

3. Bottacini F, Medini D, Pavesi A, Turroni F, Foroni E, Riley D, et al. Comparative genomics of the genus Bifidobacterium. Microbiology. 2010;156(Pt 11):3243-54

4. Ventura M, Turroni F, Lugli GA, van Sinderen D. Bifidobacteria and humans: our special friends, from ecological to genomics perspectives. J Sci Food Agric. 2014;94(2):163-8.

5. Medini D, Donati C, Tettelin H, Masignani V, Rappuoli R. The microbial pan-genome. Curr Opin Genet Dev. 2005;15(6):589-94.

6. Bottacini F, O'Connell Motherway M, Kuczynski J, O'Connell KJ, Serafini F, Duranti S, et al. Comparative genomics of the Bifidobacterium breve taxon. BMC Genomics. 2014;15:170.

7. Milani C, Duranti S, Lugli GA, Bottacini F, Strati F, Arioli S, et al. Comparative genomics of Bifidobacterium animalis subsp. lactis reveals a strict monophyletic bifidobacterial taxon. Appl Environ Microbiol. 2013;79(14):4304-15.

8. Sela DA, Mills DA. Nursing our microbiota: molecular linkages between bifidobacteria and milk oligosaccharides. Trends Microbiol. 2010;18(7):298-307.

9. Milani C, Lugli GA, Duranti S, Turroni F, Bottacini F, Mangifesta M, et al. Genomic encyclopedia of type strains of the genus Bifidobacterium. Appl Environ Microbiol. 2014;80(20):6290-302.

10. Sun Z, Zhang W, Guo C, Yang X, Liu W, Wu Y, et al. Comparative genomic analysis of 45 type strains of the genus Bifidobacterium: a snapshot of its genetic diversity and evolution. PLoS One. 2015;10(2):e0117912.

11. Ventura M, Canchaya C, Tauch A, Chandra G, Fitzgerald GF, Chater KF, et al. Genomics of Actinobacteria: tracing the evolutionary history of an ancient phylum. Microbiol Mol Biol Rev. 2007;71(3):495-548.

12. El Kaoutari A, Armougom F, Gordon JI, Raoult D, Henrissat B. The abundance and variety of carbohydrate-active enzymes in the human gut microbiota. Nat Rev Microbiol. 2013;11(7):497-504.

13. Cantarel BL, Lombard V, Henrissat B. Complex carbohydrate utilization by the healthy human microbiome. PLoS One. 2012;7(6):e28742.

14. Cronin M, Ventura M, Fitzgerald GF, van Sinderen D. Progress in genomics, metabolism and biotechnology of bifidobacteria. Int J Food Microbiol. 2011;149:4-18

15. O'Connell Motherway M, O'Driscoll J, Fitzgerald GF, Van Sinderen D. Overcoming the restriction barrier to plasmid transformation and targeted mutagenesis in Bifidobacterium breve UCC2003. Microb Biotechnol. 2009;2(3):321-32.

16. Murray NE. Type I restriction systems: sophisticated molecular machines (a legacy of Bertani and Weigle). Microbiol Mol Biol Rev. 2000;64(2):412-34.

17. Kobayashi I. Behavior of restriction-modification systems as selfish mobile elements and their impact on genome evolution. Nucleic Acids Res. 2001;29(18):3742-56.

18. Pingoud A, Fuxreiter M, Pingoud V, Wende W. Type II restriction endonucleases: structure and mechanism. Cell Mol Life Sci. 2005;62(6):685-707. 
19. Janscak P, Sandmeier U, Szczelkun MD, Bickle TA. Subunit assembly and mode of DNA cleavage of the type III restriction endonucleases EcoP1I and EcoP15l. J Mol Biol. 2001;306(3):417-31.

20. Yasui K, Kano Y, Tanaka K, Watanabe K, Shimizu-Kadota M, Yoshikawa H, et al. Improvement of bacterial transformation efficiency using plasmid artificial modification. Nucleic Acids Res. 2009;37(1):e3.

21. O'Connell Motherway M, Watson D, Bottacini F, Clark TA, Roberts RJ, Korlach $J$, et al. Identification of restriction-modification systems of Bifidobacterium animalis subsp. lactis CNCM I-2494 by SMRT sequencing and associated methylome analysis. PLoS One. 2014;9(4):e94875.

22. Dominguez W, O'Sullivan DJ. Developing an efficient and reproducible conjugation-based gene transfer system for bifidobacteria. Microbiology. 2013;159(Pt 2):328-38.

23. Bottacini F, O'Connell Motherway M, Casey E, McDonnell B, Mahony J, Ventura M, et al. Discovery of a Conjugative Megaplasmid in Bifidobacterium breve. Appl Environ Microbiol. 2015;81(1):166-76.

24. Sakaguchi K, He J, Tani S, Kano Y, Suzuki T. A targeted gene knockout method using a newly constructed temperature-sensitive plasmid mediated homologous recombination in Bifidobacterium longum. Appl Microbiol Biotechnol. 2012;95(2):499-509.

25. Hirayama Y, Sakanaka M, Fukuma H, Murayama H, Kano Y, Fukiya S, et al. Development of a Double-Crossover Markerless Gene Deletion System in Bifidobacterium longum: Functional Analysis of the a-Galactosidase Gene for Raffinose Assimilation. Appl Environ Microbiol. 2012;78(14):4984-94.

26. Sela DA, Chapman J, Adeuya A, Kim JH, Chen F, Whitehead TR, et al. The genome sequence of Bifidobacterium longum subsp. infantis reveals adaptations for milk utilization within the infant microbiome. Proc Natl Acad Sci U S A. 2008;105(48):18964-9.

27. Ventura M, Turroni F, Zomer A, Foroni E, Giubellini V, Bottacini F, et al. The Bifidobacterium dentium Bd1 genome sequence reflects its genetic adaptation to the human oral cavity. PLoS Genet. 2009;5(12):e1000785.

28. Turroni F, Bottacini F, Foroni E, Mulder I, Kim JH, Zomer A, et al. Genome analysis of Bifidobacterium bifidum PRL2010 reveals metabolic pathways for host-derived glycan foraging. Proc Natl Acad Sci U S A. 2010;107(45):19514-9.

29. Bottacini F, Milani C, Turroni F, Sanchez B, Foroni E, Duranti S, et al. Bifidobacterium asteroides PRL2011 genome analysis reveals clues for colonization of the insect gut. PLoS One. 2012;7(9):e44229.

30. Pokusaeva K, Fitzgerald GF, van Sinderen D. Carbohydrate metabolism in Bifidobacteria. Genes Nutr. 2011;6(3):285-306.

31. Lombard V, Golaconda Ramulu H, Drula E, Coutinho PM, Henrissat B. The carbohydrate-active enzymes database (CAZy) in 2013. Nucleic Acids Res. 2014;42(Database issue):D490-495.

32. O'Connell Motherway M, Zomer A, Leahy SC, Reunanen J, Bottacini F, Claesson MJ, et al. Functional genome analysis of Bifidobacterium breve UCC2003 reveals type IVb tight adherence (Tad) pili as an essential and conserved host-colonization factor. Proc Natl Acad Sci U S A. 2011;108(27):11217-22.

33. Fanning S, Hall LJ, Cronin M, Zomer A, MacSharry J, Goulding D, et al. Bifidobacterial surface-exopolysaccharide facilitates commensal-host interaction through immune modulation and pathogen protection. Proc Natl Acad Sci U S A. 2012;109(6):2108-13.

34. Turroni F, Serafini F, Foroni E, Duranti S, O'Connell Motherway M, Taverniti V, et al. Role of sortase-dependent pili of Bifidobacterium bifidum PRL2010 in modulating bacterium-host interactions. Proc Natl Acad Sci U S A. 2013;110(27):11151-6.

35. Fanning $S$, Hall $L$, van Sinderen D. Bifidobacterium breve UCC2003 surface exopolysaccharide production is a beneficial trait mediating commensal-host interaction through immune modulation and pathogen protection. Gut Microbes. 2012;3(5):420-5.

36. Ventura M, Turroni F, Lima-Mendez G, Foroni E, Zomer A, Duranti S, et al. Comparative analyses of prophage-like elements present in bifidobacterial genomes. Appl Environ Microbiol. 2009;75(21):6929-36.

37. Goessweiner-Mohr N, Arends K, Keller W, Grohmann E. Conjugative type IV secretion systems in Gram-positive bacteria. Plasmid. 2013;70(3):289-302.

38. Tettelin H, Masignani V, Cieslewicz MJ, Donati C, Medini D, Ward NL, et al. Genome analysis of multiple pathogenic isolates of Streptococcus agalactiae: implications for the microbial "pan-genome". Proc Natl Acad Sci U S A. 2005;102(39):13950-5.

39. Duranti S, Turroni F, Milani C, Foroni E, Bottacini F, Dal Bello F, et al. Exploration of the Genomic Diversity and Core Genome of the
Bifidobacterium adolescentis Phylogenetic Group by Means of a Polyphasic Approach. Appl Environ Microbiol. 2013;79(1):336-46.

40. Sakata S, Kitahara M, Sakamoto M, Hayashi H, Fukuyama M, Benno Y. Unification of Bifidobacterium infantis and Bifidobacterium suis as Bifidobacterium longum. Int J Syst Evol Microbiol. 2002;52(Pt 6):1945-51.

41. Crociani F, Alessandrini A, Mucci MM, Biavati B. Degradation of complex carbohydrates by Bifidobacterium spp. Int J Food Microbiol. 1994;24(1-2):199-210.

42. Mattarelli P, Bonaparte C, Pot B, Biavati B. Proposal to reclassify the three biotypes of Bifidobacterium longum as three subspecies: Bifidobacterium longum subsp. longum subsp. nov., Bifidobacterium longum subsp. infantis comb. nov. and Bifidobacterium longum subsp. suis comb. nov. Int J Syst Evol Microbiol. 2008;58(4):767-72.

43. Yanokura E, Oki K, Makino H, Modesto M, Pot B, Mattarelli P, et al. Subspeciation of Bifidobacterium longum by multilocus approaches and amplified fragment length polymorphism: Description of B. longum subsp. suillum subsp. nov., isolated from the faeces of piglets. Syst Appl Microbiol. 2015;38(5):305-14.

44. Wei YX, Zhang ZY, Liu C, Zhu YZ, Zhu YQ, Zheng H, et al. Complete genome sequence of Bifidobacterium longum JDM301. J Bacteriol. 2010;192(15):4076-7.

45. Yu H, Liu L, Chang Z, Wang S, Wen B, Yin P, Liu D, Chen B, Zhang J. Genome Sequence of the Bacterium Bifidobacterium longum Strain CMCC P0001, a Probiotic Strain Used for Treating Gastrointestinal Disease. Genome Announc. 2013, 1(5).

46. Ventura M, Canchaya C, Del Casale A, Dellaglio F, Neviani E, Fitzgerald GF, et al. Analysis of bifidobacterial evolution using a multilocus approach. Int J Syst Evol Microbiol. 2006;56(Pt 12):2783-92.

47. Egan $M, O^{\prime}$ Connell Motherway $M$, Ventura M, van Sinderen D. Metabolism of sialic acid by Bifidobacterium breve UCC2003. Appl Environ Microbiol. 2014;80(14):4414-26.

48. Clark TA, Lu X, Luong K, Dai Q, Boitano M, Turner SW, et al. Enhanced 5methylcytosine detection in single-molecule, real-time sequencing via Tet1 oxidation. BMC Biol. 2013;11:4.

49. Kozdon JB, Melfi MD, Luong K, Clark TA, Boitano M, Wang S, et al. Global methylation state at base-pair resolution of the Caulobacter genome throughout the cell cycle. Proc Natl Acad Sci U S A. 2013;110(48):E4658-4667.

50. Sitaraman R, Dybvig K. The hsd loci of Mycoplasma pulmonis: organization, rearrangements and expression of genes. Mol Microbiol. 1997;26(1):109-20.

51. Law J, Buist G, Haandrikman A, Kok J, Venema G, Leenhouts K. A system to generate chromosomal mutations in Lactococcus lactis which allows fast analysis of targeted genes. J Bacteriol. 1995;177(24):7011-8.

52. Fukuda STH, Hase K, Oshima K, Nakanishi Y, Yoshimura K, Tobe T, et al. Bifidobacteria can protect from enteropathogenic infection through production of acetate. Nature. 2011;469(7331):543-7.

53. Brancaccio VF, Zhurina DS, Riedel CU. Tough nuts to crack: site-directed mutagenesis of bifidobacteria remains a challenge. Bioengineered. 2013;4(4):197-202.

54. Kim JY, Wang Y, Park MS, Ji GE. Improvement of transformation efficiency through in vitro methylation and Sacll site mutation of plasmid vector in Bifidobacterium longum MG1. J Microbiol Biotechnol. 2010;20(6):1022-6.

55. De Man JC, Rogosa M, Sharpe ME. A MEDIUM FOR THE CULTIVATION OF LACTOBACILLI. J Appl Bacteriol. 1960;23(1):130-5.

56. Sambrook JFE, Maniatis T. Molecular cloning: a laboratory manual. Cold Spring Harbor Laboratory: New York; 1989.

57. Bertani G. Lysogeny at Mid-Twentieth Century: P1, P2, and Other Experimental Systems. J Bacteriol. 2004;186(3):595-600.

58. O'Riordan K. Studies on antimicrobial activity and genetic diversity of Bifidobacterium species: molecular characterisation of a $5.75 \mathrm{~kb}$ plasmid and a chromosomally encoded recA gene homologue from Bifidobacterium breve. Cork: National University of Ireland; 1998.

59. Murray IA, Clark TA, Morgan RD, Boitano M, Anton BP, Luong K, et al. The methylomes of six bacteria. Nucleic Acids Res. 2012;40(22):11450-62.

60. Fang G, Munera D, Friedman DI, Mandlik A, Chao MC, Banerjee O, et al. Genome-wide mapping of methylated adenine residues in pathogenic Escherichia coli using single-molecule real-time sequencing. Nat Biotechnol. 2012;30(12):1232-9.

61. Altschul SF, Gish W, Miller W, Myers EW, Lipman DJ. Basic local alignment search tool. J Mol Biol. 1990;215(3):403-10. 
62. Rutherford K, Parkhill J, Crook J, Horsnell T, Rice P, Rajandream MA, et al. Artemis: sequence visualization and annotation. Bioinformatics. 2000;16(10):944-5.

63. Punta M, Coggill PC, Eberhardt RY, Mistry J, Tate J, Boursnell C, et al. The Pfam protein families database. Nucleic Acids Res. 2012;40(Database issue):D290-301.

64. Tatusov RL, Fedorova ND, Jackson JD, Jacobs AR, Kiryutin B, Koonin EV, et al. The COG database: an updated version includes eukaryotes. BMC Bioinformatics. 2003;4:41.

65. Tatusov RL, Galperin MY, Natale DA, Koonin EV. The COG database: a tool for genome-scale analysis of protein functions and evolution. Nucleic Acids Res. 2000;28(1):33-6.

66. Zhao Y, Wu J, Yang J, Sun S, Xiao J, Yu J. PGAP: Pan-Genomes Analysis Pipeline. Bioinformatics. $2012 ; 28(3): 416-8$.

67. Larkin MA, Blackshields G, Brown NP, Chenna R, McGettigan PA, McWilliam $H$, et al. Clustal W and Clustal X version 2.0. Bioinformatics. 2007:23(21):2947-8.

68. Guindon S, Dufayard JF, Lefort V, Anisimova M, Hordijk W, Gascuel O. New algorithms and methods to estimate maximum-likelihood phylogenies: assessing the performance of PhyML 3.0. Syst Biol. 2010;59(3):307-21.

69. Delcher AL, Kasif S, Fleischmann RD, Peterson J, White O, Salzberg SL. Alignment of whole genomes. Nucleic Acids Res. 1999;27(11):2369-76.

70. Alvarez-Martin P, O'Connell-Motherway M, van Sinderen D, Mayo B. Functional analysis of the $\mathrm{pBC} 1$ replicon from Bifidobacterium catenulatum L48. Appl Microbiol Biotechnol. 2007;76(6):1395-402.

71. Cronin M, Knobel M, O'Connell-Motherway M, Fitzgerald GF, van Sinderen D. Molecular dissection of a bifidobacterial replicon. Appl Environ Microbiol. 2007:73(24):7858-66.

72. McGrath S, Fitzgerald GF, van Sinderen D: Improvement and optimization of two engineered phageresistance mechanisms in Lactococcus lactis. Appl Environ Microbiol. 2001;67(2):608-616.

73. Wang RF, Kushner SR. Construction of versatile low-copy-number vectors for cloning, sequencing and gene expression in Escherichia coli. Gene. 1991;100:195-9.

74. Kuipers A, de Boef E, Rink R, Fekken S, Kluskens LD, Driessen AJM, et al. NisT, the Transporter of the Lantibiotic Nisin, Can Transport Fully Modified, Dehydrated, and Unmodified Prenisin and Fusions of the Leader Peptide with Non-lantibiotic Peptides. J Biol Chem. 2004;279(21):22176-82.

\section{Submit your next manuscript to BioMed Central and take full advantage of:}

- Convenient online submission

- Thorough peer review

- No space constraints or color figure charges

- Immediate publication on acceptance

- Inclusion in PubMed, CAS, Scopus and Google Scholar

- Research which is freely available for redistribution 